\title{
UN RÉSULTAT DE CONVERGENCE D'ORDRE DEUX EN TEMPS POUR L'APPROXIMATION DES ÉQUATIONS DE NAVIER-STOKES PAR UNE TECHNIQUE DE PROJECTION INCRÉMENTALE
}

\author{
JEAn-LuC GuERMOND ${ }^{1}$
}

\begin{abstract}
The Navier-Stokes equations are approximated by means of a fractional step, ChorinTemam projection method; the time derivative is approximated by a three-level backward finite difference, whereas the approximation in space is performed by a Galerkin technique. It is shown that the proposed scheme yields an error of $\mathcal{O}\left(\delta t^{2}+h^{l+1}\right)$ for the velocity in the norm of $l^{2}\left(\mathrm{~L}^{2}(\Omega)^{d}\right)$, where $l \geq 1$ is the polynomial degree of the velocity approximation. It is also shown that the splitting error of projection schemes based on the incremental pressure correction is of $\mathcal{O}\left(\delta t^{2}\right)$ independent of the approximation order of the velocity time derivative.
\end{abstract}

Résumé. Les équations de Navier-Stokes sont approchées en temps par une schéma de différentiation rétrograde d'ordre deux et une technique de pas fractionnaire du type projection de Chorin-Temam ; l'approximation spatiale est réalisée par une technique de Galerkin. On montre qu'en temps fini, le schéma est d'ordre $\mathcal{O}\left(\delta t^{2}+h^{l+1}\right)$ pour la vitesse dans la norme $l^{2}\left(\mathrm{~L}^{2}(\Omega)^{d}\right)$, où $l \geq 1$ est le degré polynomial d'approximation de la vitesse. On montre aussi que l'erreur de fractionnement des schémas de projection basés sur une correction de pression incrémentale est d'ordre $\mathcal{O}\left(\delta t^{2}\right)$, que l'approximation de la dérivée temporelle de la vitesse soit d'ordre un ou d'ordre deux.

AMS Subject Classification. 35Q30, 65M12, 65M60.

Reçu : 12 Février 1997. Révisé : 26 Aout 1997.

\section{INTRODUCTION}

Les techniques de projection sont largement utilisées pour approcher en temps les équations de Navier-Stokes. Ces techniques introduites par Chorin [7,8] et Temam [21] sont basées sur une marche en temps fractionnaire qui sépare le problème de convection-diffusion de la contrainte d'incompressibilité. À chaque pas de temps, la vitesse déduite du sous-pas de convection-diffusion est projetée sur l'espace des champs de vecteurs à divergence nulle et à trace normale nulle à la frontière du domaine. Une analyse détaillée de la convergence en temps

Key words and phrases. Incompressible Navier-Stokes equations, Projection method, Second order approximation, Fractional-step method, Finite elements.

${ }^{1}$ Laboratoire d'Informatique pour la Mécanique et les Sciences de l'Ingénieur, CNRS, B.P. 133, 91403, Orsay, France. e-mail: guermond@limsi.fr. 
de l'algorithme original a été faite par Rannacher [19]. Il est montré dans [19] que l'erreur en temps est d'ordre $\delta t^{1 / 2}$ pour la vitesse (resp. la pression ) en norme $l^{\infty}\left(\mathrm{H}^{1}(\Omega)^{d}\right)$ (resp. $l^{\infty}\left(\mathrm{L}^{2}(\Omega)\right)$ ). Toutefois, en considérant des normes plus faibles, l'erreur sur la vitesse (resp. pression) est en $\mathcal{O}(\delta t)$ en norme $l^{\infty}\left(\mathrm{L}^{2}(\Omega)^{d}\right)$ (resp. $l^{\infty}\left(\mathrm{H}^{-1}(\Omega)\right)$ ).

De nombreuses variantes de cet algorithme ont été proposées pour en améliorer l'ordre de convergence, voir e.g. Quartapelle [17] pour une revue de ces techniques. La version qui nous intéresse ici est la version incrémentale, qui consiste à rendre explicite la pression à l'étape de convection-diffusion et à calculer l'incrément de pression à l'étape de projection. Ce schéma proposé par Goda [10] a été analysé par Van Kan [22] dans le cadre d'une approximation MAC. Il est montré dans [22] que l'erreur en temps de la version incrémentale où l'équation de convection-diffusion est approchée par un schéma de Crank-Nicolson est effectivement d'ordre deux, mais l'analyse est formelle et conduit à une majoration de l'erreur en temps par $c(h) \delta t^{2}$, où $c(h)$ est une constante qui dépend du paramètre de discrétisation spatiale et $c(h) \rightarrow+\infty$ lorsque $h \rightarrow 0$ (typiquement $\left.c(h)=\mathcal{O}\left(1 / h^{2}\right)\right)$.

Dans cet article on analyse un schéma de projection incrémental basé sur une approximation par différentiation rétrograde d'ordre deux pour la dérivée en temps et une technique de Galerkin pour l'approximation en espace. L'analyse de convergence en temps et en espace du schéma fournit une erreur en $c\left(\delta t^{2}+h^{l+1}\right)$ pour la vitesse en norme $l^{2}\left(\mathrm{~L}^{2}(\Omega)^{d}\right)$, où la constante $c$ ne depend ni de $h$ ni de $\delta t$. L'entier $l \geq 1$ est le degré polynomial d'approximation de la vitesse. En norme $l^{\infty}\left(\mathrm{L}^{2}(\Omega)^{d}\right)$ on donne une estimation en $\mathcal{O}\left(\delta t^{7 / 4}+h^{l+1}\right)$. Cet article est divisé en quatre parties. Les notations, les définitions et l'algorithme de projection sont présentés dans la section 2. Des résultats préliminaires de convergence à l'ordre un en temps sont donnés dans la section 3 . Le résultat principal de convergence à l'ordre deux en temps est énoncé et démontré dans la section 4 . On donne finalement dans la section 5 une estimation de l'erreur de fractionnement pour le schéma basé sur l'approximation d'Euler rétrograde. Le résultat remarquable de cette section est que l'erreur de fractionnement est d'ordre deux (dans les normes appropriées) alors que le schéma est d'ordre un.

\section{Position DU PROBLÈme}

\subsection{Hypothèses et notations}

Soit $\Omega$ un ouvert borné et connexe de $\mathbb{R}^{d}(d \leq 3)$ dont la frontière est régulière. Plus précisement on suppose que $\Omega$ est suffisament régulier pour que l'opérateur de Stokes possède les propriétés de régularisation habituelles ( $c f$. les estimations de Cattabriga [6] ou Amrouche et Girault [1]).

Le domaine $\Omega$ est supposé rempli d'un fluide incompressible obéissant aux équations de Navier-Stokes

$$
\mathcal{P}\left\{\begin{array}{l}
\frac{\partial u}{\partial t}-\Delta u+(u \cdot \nabla) u+\nabla p=f \quad \text { dans } \Omega \times(0, T), \\
\nabla \cdot u=0 \quad \text { dans } \Omega \times(0, T), \\
u=0 \quad \text { sur } \partial \Omega \times(0, T), \\
u=u_{0} \quad \text { sur } \Omega \times\{0\},
\end{array}\right.
$$

où $f$ est un champ de force donné et $u_{0}$ est un champ de vecteur solénoidal à trace normale nulle.

Par la suite on adopte les notations habituelles pour les espaces de Sobolev réels : $W^{s, p}(\Omega), 0 \leq s<\infty$, $0 \leq p \leq \infty$ et on note $\|\cdot\|_{s, p}$ et $|\cdot|_{s, p}$ les normes et semi-normes correspondantes. Le complété pour la norme $\|\cdot\|_{s, p}$ de l'espace des fonctions $C^{\infty}$ à support compact dans $\Omega$ est noté $W_{0}^{s, p}(\Omega)$. Pour simplifier, l'espace de Hilbert $W^{s, 2}(\Omega)\left(\right.$ resp. $\left.W_{0}^{s, p}(\Omega)\right)$ est noté $H^{s}(\Omega)$ (resp. $\left.H_{0}^{s}(\Omega)\right)$; les normes et semi-normes correspondantes sont notées $\|\cdot\|_{s}$ et $|\cdot|_{s}$; le dual de $H_{0}^{s}(\Omega)$ est noté $H^{-s}(\Omega)$. Le sous-espace de $\mathrm{L}^{2}(\Omega)$ des fonctions à moyenne nulle est noté $\mathrm{L}_{0}^{2}(\Omega)$. 


\subsection{L'approximation spatiale}

Afin de construire une approximation de Galerkin de (2.1) on introduit $\left(X_{h}, M_{h}\right)$ une approximation mixte de $\left(\mathrm{H}_{0}^{1}(\Omega)^{d}, \mathrm{~L}_{0}^{2}(\Omega)\right)$. On suppose par la suite que cette approximation vérifie les propriétés suivantes (cf. e.g. Bernardi-Raugel [3], Girault-Raviart [9], ou bien Quarteroni-Valli [18]) :

Il existe $l \in \mathbb{N}, l \geq 1$ et $c>0$ tels que pour tout $r \in[0, l]$

$$
\left\{\begin{array}{l}
\inf _{v_{h} \in X_{h}}\left\{\left\|v-v_{h}\right\|_{0}+h\left\|v-v_{h}\right\|_{1}\right\} \leq c h^{r+1}\|v\|_{r+1}, \quad \forall v \in \mathrm{H}^{r+1}(\Omega)^{d} \cap \mathrm{H}_{0}^{1}(\Omega)^{d}, \\
\inf _{v_{h} \in X_{h}}\left\|v-v_{h}\right\|_{1, p} \leq c h^{r}\|v\|_{r+1, p}, \quad 2 \leq p \leq \infty, \quad \forall v \in W^{r+1, p}(\Omega)^{d} \cap \mathrm{H}_{0}^{1}(\Omega)^{d} .
\end{array}\right.
$$

Il existe $c>0$ tel que pour tout $r \in[0, l]$ et tout $q$ in $H^{r}(\Omega) \cap \mathrm{L}_{0}^{2}(\Omega)$,

$$
\inf _{q_{h} \in M_{h}}\left\|q-q_{h}\right\|_{0} \leq c h^{r}\|q\|_{r}
$$

Enfin, il existe $c>0$ tel que pour tout $v_{h}$ dans $X_{h}$, on a :

$$
\begin{gathered}
\left\|v_{h}\right\|_{n, p} \leq c h^{m-n+\frac{d}{p}-\frac{d}{q}}\left\|v_{h}\right\|_{m, q}, \quad 0 \leq m \leq n \leq 1, \quad 1 \leq q \leq p \leq \infty . \\
\left\{\begin{array}{l}
\left\|v_{h}\right\|_{0, \infty} \leq c\left(1+\left|\log h^{-1}\right|\right)^{1 / 2}\left\|v_{h}\right\|_{1,2}, \quad \text { en dimension 2, } \\
\left\|v_{h}\right\|_{0, \infty} \leq c h^{-1 / 2}\left\|v_{h}\right\|_{1,2}, \quad \text { en dimension 3. }
\end{array}\right.
\end{gathered}
$$

Remarque 2.1. Les éléments finis $P_{l} / P_{l-1}$ avec $2 \leq l$ satisfont ces hypothèses. L'entier $l$ est le degré polynomial de l'approximation de la vitesse et la pression est approchée par des polynomes de degré $l-1$. Pour les éléments finis $P_{1}$-iso- $P_{2} / P_{1}$ et $P_{1}$-bulle $/ P_{1}$ ces hypothèses sont vérifiées avec $l=1 ; c f$. [9] pour d'autres exemples.

Afin de simplifier l'écriture des formulations variationnelles, on introduit le laplacien discret $A_{h}: X_{h} \longrightarrow X_{h}^{\prime}$ tel que pour tout $\left(u_{h}, v_{h}\right) \in X_{h} \times X_{h},\left(A_{h} u_{h}, v_{h}\right)=\left(\nabla u_{h}, \nabla v_{h}\right)$. De plus on note $\pi_{h}: \mathrm{H}^{-1}(\Omega)^{d} \longrightarrow X_{h}^{\prime}$ l'opérateur tel que $\left(\pi_{h} f, v_{h}\right)=\left\langle f, v_{h}\right\rangle$ pour tout $f \in \mathrm{H}^{-1}(\Omega)^{d}$ et tout $v_{h} \in X_{h}$.

Pour représenter le terme de convection on introduit la forme trilinéaire :

$$
\forall(u, v, w) \in\left[\mathrm{H}_{0}^{1}(\Omega)^{d}\right]^{3}, \quad d(u, v, w)=((u \cdot \nabla) v, w)+\frac{1}{2}(\nabla \cdot u, v \cdot w),
$$

où $u \cdot v$ désigne le produit scalaire euclidien dans $\mathbb{R}^{d}$. Cette forme trilinéaire coïncides avec la forme habituelle $(u \cdot \nabla v, w)$ lorsque $u$ est à divergence nulle. En utilisant l'inégalité de Hölder et quelques inégalités de Sobolev classiques ( $c f$. eg. Brezis [4]), on obtient les inégalités suivantes, souvent utilisées par la suite :

$$
\left\{\begin{array}{l}
\max (d(u, v, w), d(v, u, w)) \leq c\left(\|u\|_{0, \infty}+\|u\|_{1,3}\right)\|v\|_{0,2}\|w\|_{1,2}, \\
\max (d(u, v, w), d(v, u, w)) \leq c\|u\|_{0,2}\left(\|v\|_{0, \infty}\|w\|_{1,2}+\|v\|_{1,2}\|w\|_{0, \infty}\right) .
\end{array}\right.
$$

Rappellons que les inégalités d'interpolation de Gagliardo-Nirenberg [4] fournissent la majoration très utile :

$$
\|u\|_{0, \infty}+\|u\|_{1,3} \leq c\|u\|_{1,2}^{1 / 2}\|u\|_{2,2}^{1 / 2} .
$$

L'opérateur de convection discret $D_{h}: X_{h} \times X_{h} \longrightarrow X_{h}^{\prime}$ est défini par $\left(D_{h}\left(u_{h}, v_{h}\right), w_{h}\right)=d\left(u_{h}, v_{h}, w_{h}\right)$. La forme trilinéaire $d$ étant anti-symétrique par rapport à la deuxième et la troisième variable $(d(u, v, w)=-d(u, w, v))$, 
le produit scalaire $\left(D_{h}\left(u_{h}, v_{h}\right), v_{h}\right)$ est nécessairement nul ; l'opérateur $D_{h}\left(u_{h}, \cdot\right)$ est donc monotone. Cette propriété reproduit au niveau discret une propriété bien connue de la forme continue dès que $u$ est à divergence nulle et à trace normale nulle.

Enfin, on introduit la divergence discrète $B_{h}: X_{h} \longrightarrow M_{h}$ et le gradient discret (l'opérateur transposé) $B_{h}^{\mathrm{t}}: M_{h} \longrightarrow X_{h}^{\prime}$ de telle sorte que pour tout couple $\left(v_{h}, q_{h}\right)$ de $X_{h} \times M_{h}$ on ait $\left(B_{h} v_{h}, q_{h}\right)=\left(v_{h}, B_{h}^{\mathrm{t}} q_{h}\right)=$ $-\left(\nabla \cdot v_{h}, q_{h}\right)$. On suppose par la suite que $B_{h}$ est surjectif ; c'est-à-dire que l'approximation mixte vérifie la condition de Babǔska-Brezzi $[2,5]$ :

$$
\exists c>0, \quad \inf _{q_{h} \in M_{h}} \sup _{v_{h} \in X_{h}} \frac{\left(B_{h} v_{h}, q_{h}\right)}{\left\|v_{h}\right\|_{1}\left\|q_{h}\right\|_{0}} \geq c .
$$

Pour découpler la contrainte d'incompressibilité du problème d'évolution temporelle, on introduit comme dans Guermond [11,12] un prolongement de $B_{h}$. On se donne $Y_{h}$ un sous-espace de dimension finie de $\mathrm{L}^{2}(\Omega)^{d}$ et on suppose que $X_{h} \subset Y_{h}$. L'injection de $X_{h}$ dans $Y_{h}$ est notée $i_{h}$; le transposé de $i_{h}$ est la projection $\mathrm{L}^{2}$ de $Y_{h}$ sur $X_{h}$. On suppose que $Y_{h}$ et $M_{h}$ sont compatibles au sens suivant : ou bien $Y_{h} \subset \mathrm{H}_{0}^{d i v}(\Omega)$ ou bien $M_{h} \subset \mathrm{H}^{1}(\Omega)$. Par exemple, le choix trivial est $Y_{h}=X_{h}$, mais on peut aussi poser $Y_{h}=X_{h}+\nabla M_{h}$ dès que $M_{h} \subset \mathrm{H}^{1}(\Omega)($ le lecteur est renvoyé à Guermond [11] pour une revue détaillée des différents choix de $\left.Y_{h}\right)$. On définit maintenant $C_{h}: Y_{h} \longrightarrow M_{h}$ tel que pour tout $\left(v_{h}, q_{h}\right)$ dans $Y_{h} \times M_{h}$, ou bien $\left(C_{h} v_{h}, q_{h}\right)=-\left(\nabla \cdot v_{h}, q_{h}\right)$ si $Y_{h} \subset \mathrm{H}_{0}^{\text {div }}(\Omega)$ ou $\left(C_{h} v_{h}, q_{h}\right)=\left(v_{h}, \nabla q_{h}\right)$ si $M_{h} \subset \mathrm{H}^{1}(\Omega)$. On montre alors que $C_{h}$ est un prolongement de $B_{h}$ :

$$
C_{h} i_{h}=B_{h}, \quad i_{h}^{\mathrm{t}} C_{h}^{\mathrm{t}}=B_{h}^{\mathrm{t}}
$$

$C_{h}$ est surjectif car c'est le prolongement d'un opérateur surjectif. Par la suite on note $H_{h}=\operatorname{ker} C_{h}, V_{h}=\operatorname{ker} B_{h}$ et on introduit $P_{H_{h}}: Y_{h} \longrightarrow H_{h}$ la projection $\mathrm{L}^{2}$ de $Y_{h}$ sur $H_{h}$. L'opérateur $C_{h}$ possède la propriété de stabilité suivante

Proposition 2.1. Soient $q_{h} \in M_{h}, q \in \mathrm{H}^{1}(\Omega)$ et $c_{0}>0$ tels que $\left\|q-q_{h}\right\|_{0} \leq c_{0} h\|q\|_{1}$; alors il existe $c\left(c_{0}\right)>0$ tel que $\left\|C_{h}^{\mathrm{t}} q_{h}\right\|_{0} \leq c\left(c_{0}\right)\|q\|_{1}$.

\subsection{L'algorithme de projection}

Pour un temps fini $T>0$ donné et un entier $K \geq 2$, on définit $\delta t=T / K$ et on introduit $t^{0}, t^{1}, \ldots, t^{K}$ une partition de l'intervalle $[0, T]$ de telle sorte que $t^{k}=k \delta t$ pour $0 \leq k \leq K$. Pour toute fonction continue du temps, $\phi(t)$, on note $\phi^{k}=\phi\left(t^{k}\right)$ et on introduit l'opérateur $\delta_{t} \phi^{k}=\phi^{k}-\phi^{k-1}$ et la convention $\delta_{t t} \phi^{k}=\delta_{t}\left(\delta_{t} \phi\right)^{k}$.

On construit maintenant deux suites de vitesses approchées $\left\{\tilde{u}_{h}^{k} \in X_{h}\right\},\left\{u_{h}^{k} \in Y_{h}\right\}$ et une suite de pressions approchées $\left\{p_{h}^{k} \in M_{h}\right\}$. On suppose données $\hat{u}_{h}^{0} \in V_{h}, \hat{u}_{h}^{1} \in V_{h}$ et $\hat{p}_{h}^{1} \in M_{h}$ des approximations de $u_{\mid t=0}$, $u_{\mid t=\delta t}$ et $p_{\mid t=\delta t}$. Noter que $p_{\mid t=\delta t}$ n'est pas une donnée mais se déduit de $u_{\mid t=\delta t}$ dès que $u(t)$ est suffisamment régulier au voisinage de $t=0$. On suppose que toutes les conditions de compatibilité requises par la régularité demandée sont satisfaites ( $c f$. eg. Heywood-Rannacher [16] pour les détails sur cette question, et $c f$. GuermondQuartapelle $[13,14]$ pour une technique alternative si la régularitée en $t=0$ n'est pas satisfaite). On peut obtenir $\hat{u}_{h}^{1}$ et $\hat{p}_{h}^{1}$ par différentes techniques (un pas de Crank-Nicolson, un pas de Runge-Kutta implicite, ...) dont on ne discutera pas ici.

Pour $k=0,1$ on pose $u_{h}^{k}=\tilde{u}_{h}^{k}=\hat{u}_{h}^{k}$ et pour $k=1$ on pose $p_{h}^{1}=\hat{p}_{h}^{1}$. Pour $1 \leq k \leq K-1$ on note $f_{h}^{k+1}=\pi_{h} f^{k+1}$ et on cherche $\tilde{u}_{h}^{k+1}$ dans $X_{h}$ tel que :

$$
\frac{3 \tilde{u}_{h}^{k+1}-4 i_{h}^{\mathrm{t}} u_{h}^{k}+i_{h}^{\mathrm{t}} u_{h}^{k-1}}{2 \delta t}+A_{h} \tilde{u}_{h}^{k+1}+D_{h}\left(2 \tilde{u}_{h}^{k}-\tilde{u}_{h}^{k-1}, \tilde{u}_{h}^{k+1}\right)=f_{h}^{k+1}-B_{h}^{\mathrm{t}} p_{h}^{k}
$$


On cherche ensuite $u_{h}^{k+1}$ dans $Y_{h}$ et $p_{h}^{k+1}$ dans $M_{h}$ tel que

$$
\left\{\begin{array}{l}
\frac{3 u_{h}^{k+1}-3 i_{h} \tilde{u}_{h}^{k+1}}{2 \delta t}+C_{h}^{\mathrm{t}}\left(p_{h}^{k+1}-p_{h}^{k}\right)=0 \\
C_{h} u_{h}^{k+1}=0
\end{array}\right.
$$

Remarque 2.2. En pratique les vitesses projetées $u_{h}^{k}$ et $u_{h}^{k-1}$ sont éliminées de l'algorithme en remplacant leurs valeurs déduites de l'étape de projection (2.12) et en utilisant la relation $i_{h}^{\mathrm{t}} C_{h}^{\mathrm{t}}=B_{h}^{\mathrm{t}}(c f$. [11-14]). Pour $k \geq 3$, la forme variationnelle de l'étape de convection-diffusion s'écrit

$$
\begin{array}{r}
\left(\frac{3 \tilde{u}_{h}^{k+1}-4 \tilde{u}_{h}^{k}+\tilde{u}_{h}^{k-1}}{2 \delta t}, v_{h}\right)+\left(\nabla \tilde{u}_{h}^{k+1}, \nabla v_{h}\right)+d\left(2 \tilde{u}_{h}^{k}-\tilde{u}_{h}^{k-1}, \tilde{u}_{h}^{k+1}, v_{h}\right) \\
-\frac{1}{3}\left(7 p_{h}^{k}-5 p_{h}^{k-1}+p_{h}^{k-2}, \nabla \cdot v_{h}\right)=\left(f^{k+1}, v_{h}\right), \quad \forall v_{h} \in X_{h},
\end{array}
$$

et l'étape de projection se réduit à la détermination de la pression en résolvant :

$$
C_{h} C_{h}^{\mathrm{t}}\left(p_{h}^{k+1}-p_{h}^{k}\right)=\frac{3 B_{h} \tilde{u}_{h}^{k+1}}{2 \delta t} .
$$

Pour le calcul de $\tilde{u}_{h}^{3}$ il suffit d'éliminer $u_{h}^{2}$ et pour le calcul de $\tilde{u}_{h}^{2}$ aucune vitesse n'est à éliminer puisque $u_{h}^{1}$ et $u_{h}^{0}$ sont connues. Remarquer qu'à l'étape de convection-diffusion le terme de pression $\left(7 p_{h}^{k}-5 p_{h}^{k-1}+p_{h}^{k-2}\right) / 3$ peut s'écrire $2 p_{h}^{k}-p_{h}^{k-1}+\left(p_{h}^{k}-2 p_{h}^{k-1}+p_{h}^{k-2}\right) / 3$; c'est-à-dire que ce terme est formellement une extrapolation d'ordre deux.

Remarque 2.3. Si on choisit $M_{h} \subset \mathrm{H}^{1}(\Omega)$ et $Y_{h}=X_{h}+\nabla M_{h}$, alors $C_{h}^{\mathrm{t}}$ est la restriction de $\nabla$ à $M_{h}(c f$. [11-14]) et le calcul de la pression à l'étape de projection s'écrit sous la forme variationnelle classique suivante : trouver $p_{h}^{k+1}$ dans $M_{h}$ tel que

$$
\left(\nabla\left(p_{h}^{k+1}-p_{h}^{k}\right), \nabla q_{h}\right)=-\frac{3\left(\nabla \cdot \tilde{u}_{h}^{k+1}, q_{h}\right)}{2 \delta t}, \quad \forall q_{h} \in M_{h}
$$

et $u_{h}^{k+1}$ est directement donné par

$$
u_{h}^{k+1}=\tilde{u}_{h}^{k+1}-\frac{2 \delta t}{3} \nabla\left(p_{h}^{k+1}-p_{h}^{k}\right)
$$

\section{Estimations D'ORDRE Un EN TEMPS}

\subsection{Préliminaires}

Pour un espace de Banach donné $W$ on note $L^{p}(W)$ l'espace $L^{p}(0, T ; W)$. On note $l^{p}(W)$ l'espace $\left\{\left(w^{0}, \ldots, w^{K}\right) ; w^{k} \in W, 0 \leq k \leq K\right\}$ muni de la norme :

$$
\left\{\begin{aligned}
\|w\|_{l^{p}(W)} & =\left(\delta t \sum_{k=0}^{K}\left\|w^{k}\right\|_{W}^{p}\right)^{1 / p}, \quad \text { pour } 1 \leq p<\infty, \\
\|w\|_{l^{\infty}(W)} & =\max _{0 \leq k \leq K}\left\|w^{k}\right\|_{W} .
\end{aligned}\right.
$$


On définit une approximation de $u(t)$ et $p(t)$ comme suit. Pour tout $t \in[0, T]$, on note $\left(w_{h}(t), q_{h}(t)\right) \in X_{h} \times M_{h}$ la solution du problème de Stokes discret :

$$
\left\{\begin{array}{l}
\left(\nabla w_{h}(t), \nabla v_{h}\right)+\left(B_{h}^{\mathrm{t}} q_{h}(t), v_{h}\right)=\left(\nabla u(t), \nabla v_{h}\right)-\left(p(t), \nabla \cdot v_{h}\right), \quad \forall v_{h} \in X_{h}, \\
\left(B_{h} w_{h}(t), r_{h}\right)=-\left(\nabla \cdot u(t), r_{h}\right), \quad \forall r_{h} \in M_{h} .
\end{array}\right.
$$

Grâce aux propriétés régularisantes de l'opérateur de Stokes dans les domaines réguliers, on montre l'estimation suivante :

Lemme 3.1. Si $u^{(j)} \in L^{\beta}\left(H^{l+1}(\Omega)^{d} \cap \mathrm{H}_{0}^{1}(\Omega)^{d}\right), p^{(j)} \in L^{\beta}\left(H^{l}(\Omega)\right)$ pour $1 \leq \beta \leq \infty$ et $j=0,1, \ldots$, alors il existe $c>0$ tel que

$$
\begin{gathered}
\left\|u^{(j)}-w_{h}^{(j)}\right\|_{L^{\beta}\left(\mathrm{L}^{2}(\Omega)^{d}\right)}+h\left[\left\|u^{(j)}-w_{h}^{(j)}\right\|_{L^{\beta}\left(\mathrm{H}^{1}(\Omega)^{d}\right)}+\left\|p^{(j)}-q_{h}^{(j)}\right\|_{L^{\beta}\left(\mathrm{L}^{2}(\Omega)\right)}\right] \\
\leq c h^{l+1}\left[\left\|u^{(j)}\right\|_{L^{\beta}\left(H^{l+1}(\Omega)^{d}\right)}+\left\|p^{(j)}\right\|_{L^{\beta}\left(H^{l}(\Omega)\right)}\right] .
\end{gathered}
$$

On a aussi le lemme suivant :

Lemme 3.2. Si pour $j$ entier $1 \leq \beta \leq \infty, u^{(j)} \in L^{\beta}\left(H^{2}(\Omega)^{d} \cap \mathrm{H}_{0}^{1}(\Omega)^{d}\right)$ et $p^{(j)} \in L^{\beta}\left(\mathrm{H}^{1}(\Omega)\right)$, alors il existe $c>0$ tel que

$$
\begin{aligned}
\left\|w_{h}^{(j)}\right\|_{L^{\beta}\left(W^{0, \infty}(\Omega)^{d} \cap W^{1,3}(\Omega)^{d}\right)} & +\left\|C_{h}^{\mathrm{t}} q_{h}^{(j)}\right\|_{L^{\beta}\left(\mathrm{L}^{2}(\Omega)\right)} \\
& \leq c\left[\left\|u^{(j)}\right\|_{L^{\beta}\left(H^{2}(\Omega)^{d}\right)}+\left\|p^{(j)}\right\|_{L^{\beta}\left(H^{1}(\Omega)\right)}\right] .
\end{aligned}
$$

\subsection{Estimation d'ordre un sur la vitesse}

Par la suite on utilise les notations suivantes pour représenter les erreurs :

$$
\left\{\begin{aligned}
\eta(t) & =u(t)-w_{h}(t), \quad \mu(t)=p(t)-q_{h}(t), \\
e_{h}^{k} & =w_{h}\left(t^{k}\right)-u_{h}^{k}, \quad \tilde{e}_{h}^{k}=w_{h}\left(t^{k}\right)-\tilde{u}_{h}^{k}, \\
\epsilon_{h}^{k} & =q_{h}\left(t^{k}\right)-p_{h}^{k} .
\end{aligned}\right.
$$

Les fonctions $\eta(t)$ et $\mu(t)$ se comportent comme des erreurs d'interpolation alors que les fonctions $e_{h}^{k}, \tilde{e}_{h}^{k}$ et $\epsilon_{h}^{k}$ sont des erreurs d'approximation. On fait les hypothèses suivantes :

(H1) $\max \left(\left\|e_{h}^{0}\right\|_{0},\left\|e_{h}^{1}\right\|_{0}\right) \leq c h^{l+1}, \quad\left\|\epsilon_{h}^{1}\right\|_{1} \leq c$.

(H2) $u$ est dans $W^{1, \infty}\left(\mathrm{H}_{0}^{1}(\Omega)^{d} \cap H^{l+1}(\Omega)^{d}\right)$, $u_{t t} \in L^{\infty}\left(\mathrm{L}^{2}(\Omega)^{d}\right), u_{t t t} \in L^{\infty}\left(\mathrm{H}^{1}(\Omega)^{d}\right)$, $p$ est dans $W^{1, \infty}\left(H^{l}(\Omega)\right)$, $p_{t t t} \in L^{\infty}\left(L^{2}(\Omega)\right)$.

Remarque 3.1. En fait, pour obtenir l'ordre 1 en temps, les hypothèses sur les dérivées troisièmes sont inutiles, on pourrait les remplacer par $u_{t t} \in L^{2}\left(\mathrm{H}^{1}(\Omega)^{d}\right)$ et $p_{t t} \in L^{2}\left(L^{2}(\Omega)\right)$. 
Un premier résultat de convergence est donné par

Théorème 3.1. Dans le cadre des hypothèses (H1-H2), la solution de l'algorithme de projection (2.11)-(2.12) satisfait les estimations :

$$
\begin{gathered}
\left\|u-u_{h}\right\|_{1^{\infty}\left(\mathrm{L}^{2}(\Omega)^{d}\right)}+\left\|u-\tilde{u}_{h}\right\|_{1^{\infty}\left(\mathrm{L}^{2}(\Omega)^{d}\right)} \leq c\left(h^{l+1}+\delta t\right), \\
\left\|u-\tilde{u}_{h}\right\|_{1^{2}\left(\mathrm{H}^{1}(\Omega)^{d}\right)} \leq c\left(h^{l}+\delta t\right) .
\end{gathered}
$$

Preuve. La preuve s'inspire des mêmes idées que dans Guermond-Quartapelle [15], on ne rappelle donc ici que les étapes spécifiques à la différentiation rétrograde d'orde deux.

Étape 1. On établit d'abord les équations qui contrôlent les erreurs. L'approximation $\left(w_{h}(t), q_{h}(t)\right)$ de la solution du problème (2.1) satisfait au temps $t^{k+1}$ l'équation :

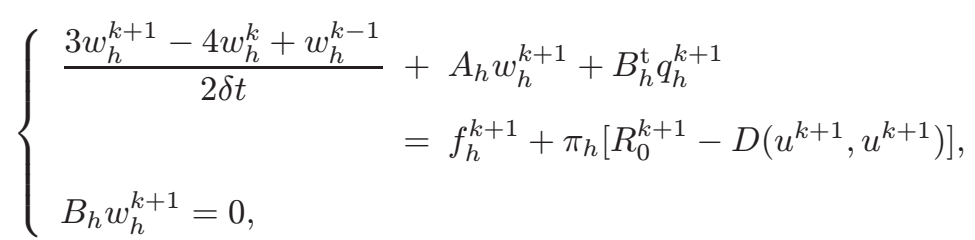

où la forme linéaire $D(u, v)$ est définie $\operatorname{par}\langle D(u, v), w\rangle=d(u, v, w)$ et le reste $R_{0}^{k+1}$ est défini par

$$
R_{0}^{k+1}=\frac{3 w_{h}^{k+1}-4 w_{h}^{k}+w_{h}^{k-1}}{2 \delta t}-u_{t}^{k+1} .
$$

On obtient l'équation contrôlant $\tilde{e}_{h}^{k+1}$ en soustrayant (2.11) à (3.8) :

$$
\frac{3 \tilde{e}_{h}^{k+1}-4 i_{h}^{\mathrm{t}} e_{h}^{k}+i_{h}^{\mathrm{t}} e_{h}^{k-1}}{2 \delta t}+A_{h} \tilde{e}_{h}^{k+1}=-B_{h}^{\mathrm{t}} \psi_{h}^{k}+\pi_{h}\left[R_{0}^{k+1}+R_{n l}^{k+1}\right]
$$

où on a posé $\psi_{h}^{k}=q_{h}^{k+1}-p_{h}^{k}=\delta_{t} q_{h}^{k+1}+\epsilon_{h}^{k}$ et le reste non linéaire est défini par

$$
\left\langle R_{n l}^{k+1}, v_{h}\right\rangle=-d\left(u^{k+1}, u^{k+1}, v_{h}\right)+d\left(2 \tilde{u}_{h}^{k}-\tilde{u}_{h}^{k-1}, \tilde{u}_{h}^{k+1}, v_{h}\right) .
$$

D'autre part, en utilisant le fait que $w_{h}^{k+1}$ est dans $X_{h}, B_{h} w_{h}^{k+1}=0$ et $C_{h}$ est un prolongement de $B_{h}$, on obtient le système qui contrôle $e_{h}^{k+1}$ et $\epsilon_{h}^{k+1}$

$$
\left\{\begin{array}{l}
\frac{3 e_{h}^{k+1}-3 i_{h} \tilde{e}_{h}^{k+1}}{2 \delta t}+C_{h}^{\mathrm{t}}\left(\epsilon_{h}^{k+1}-\psi_{h}^{k}\right)=0 \\
C_{h} e_{h}^{k+1}=0
\end{array}\right.
$$

Étape 2. On multiplie (3.9) par la fonction test $4 \delta t \tilde{e}_{h}^{k+1}$. En utilisant la relation :

$$
\begin{gathered}
2\left(a^{k+1}, 3 a^{k+1}-4 a^{k}+a^{k-1}\right)=\left|a^{k+1}\right|^{2}+\left|2 a^{k+1}-a^{k}\right|^{2}+\left|\delta_{t t} a_{k+1}\right|^{2} \\
-\left|a^{k}\right|^{2}-\left|2 a^{k}-a^{k-1}\right|^{2},
\end{gathered}
$$


pour le premier terme on déduit :

$$
\begin{aligned}
2\left(\tilde{e}_{h}^{k+1}, 3 \tilde{e}_{h}^{k+1}-4 i_{h}^{\mathrm{t}} e_{h}^{k}+i_{h}^{\mathrm{t}} e_{h}^{k-1}\right)= & 2\left(\tilde{e}_{h}^{k+1}, 3 e_{h}^{k+1}-4 e_{h}^{k}+e_{h}^{k-1}\right)+6\left(\tilde{e}_{h}^{k+1}, \tilde{e}_{h}^{k+1}-e_{h}^{k+1}\right) \\
= & 2\left(\tilde{e}_{h}^{k+1}-e_{h}^{k+1}, 3 e_{h}^{k+1}-4 e_{h}^{k}+e_{h}^{k-1}\right) \\
& +2\left(e_{h}^{k+1}, 3 e_{h}^{k+1}-4 e_{h}^{k}+e_{h}^{k-1}\right) \\
& +3\left\|\tilde{e}_{h}^{k+1}\right\|_{0}^{2}+3\left\|\tilde{e}_{h}^{k+1}-e_{h}^{k+1}\right\|_{0}^{2}-3\left\|e_{h}^{k+1}\right\|_{0}^{2} \\
= & \left\|e_{h}^{k+1}\right\|_{0}^{2}+\left\|2 e_{h}^{k+1}-e_{h}^{k}\right\|_{0}^{2}+\left\|\delta_{t t} e_{h}^{k+1}\right\|_{0}^{2} \\
& -\left\|e_{h}^{k}\right\|_{0}^{2}-\left\|2 e_{h}^{k}-e_{h}^{k-1}\right\|_{0}^{2} \\
& +3\left\|\tilde{e}_{h}^{k+1}\right\|_{0}^{2}+3\left\|\tilde{e}_{h}^{k+1}-e_{h}^{k+1}\right\|_{0}^{2}-3\left\|e_{h}^{k+1}\right\|_{0}^{2},
\end{aligned}
$$

où on a utilisé le fait que $\tilde{e}_{h}^{k+1}-e_{h}^{k+1}$ est dans $\operatorname{im}\left(C_{h}^{\mathrm{t}}\right)$ et $3 e_{h}^{k+1}-4 e_{h}^{k}+e_{h}^{k-1}$ est dans $\operatorname{ker}\left(C_{h}\right)$ par construction. En notant $\alpha$ la constante telle que $\alpha\|v\|_{1}^{2} \leq\|\nabla v\|_{0}^{2}$ pour tout $v$ dans $\mathrm{H}_{0}^{1}(\Omega)^{d}$, on obtient l'inégalité :

$$
\begin{aligned}
-2\left\|e_{h}^{k+1}\right\|_{0}^{2}+\left\|2 e_{h}^{k+1}-e_{h}^{k}\right\|_{0}^{2}+ & \left\|\delta_{t e} e_{h}^{k+1}\right\|_{0}^{2}+3\left\|\tilde{e}_{h}^{k+1}\right\|_{0}^{2}+3\left\|\tilde{e}_{h}^{k+1}-e_{h}^{k+1}\right\|_{0}^{2}+4 \alpha \delta t\left\|\tilde{e}_{h}^{k+1}\right\|_{1}^{2} \\
\leq & \left\|e_{h}^{k}\right\|_{0}^{2}+\left\|2 e_{h}^{k}-e_{h}^{k-1}\right\|_{0}^{2}-4 \delta t\left(\tilde{e}_{h}^{k+1}, B_{h}^{\mathrm{t}} \psi_{h}^{k}\right) \\
& +4 \delta t\left\langle R_{0}^{k+1}+R_{n l}^{k+1}, \tilde{e}_{h}^{k+1}\right\rangle .
\end{aligned}
$$

Le contôle du residu est classique :

$$
\begin{aligned}
4 \delta t\left\langle R_{0}^{k+1}+R_{n l}^{k+1}, \tilde{e}_{h}^{k+1}\right\rangle \leq & \gamma\left\|\tilde{e}_{h}^{k+1}\right\|_{1}^{2}+c_{1} \delta t\left(\delta t^{2}+h^{l+1}\right)^{2} \\
& +c_{2} \delta t\left(\left\|\tilde{e}_{h}^{k}-e_{h}^{k}\right\|_{0}^{2}+\left\|\tilde{e}_{h}^{k-1}-e_{h}^{k-1}\right\|_{0}^{2}+\left\|e_{h}^{k}\right\|_{0}^{2}+\left\|e_{h}^{k-1}\right\|_{0}^{2}\right),
\end{aligned}
$$

où $\gamma$ est une constante qu'on peut choisir suffisamment petite ; les constantes $c_{1}$ et $c_{2}$ dépendent linéairement de $1 / \gamma,\|u\|_{L^{\infty}\left(H^{l+1}\right)},\left\|u_{t}\right\|_{L^{\infty}\left(H^{l+1}\right)},\|p\|_{L^{\infty}\left(H^{l}\right)},\left\|p_{t}\right\|_{L^{\infty}\left(H^{l}\right)},\left\|u_{t t}\right\|_{L^{\infty}\left(L^{2}\right)},\left\|u_{t t t}\right\|_{L^{\infty}\left(H^{1}\right)}$ et $\left\|p_{t t t}\right\|_{L^{\infty}\left(L^{2}\right)}$.

On obtient un contrôle sur $4 \delta t\left(\tilde{e}_{h}^{k+1}, B_{h}^{\mathrm{t}} \psi_{h}^{k}\right)$ en multipliant (3.10) par $4 \delta t C_{h}^{\mathrm{t}} \psi_{h}^{k}$.

$$
\begin{aligned}
4 \delta t\left(\tilde{e}_{h}^{k+1}, B_{h}^{\mathrm{t}} \psi_{h}^{k}\right)= & \frac{4}{3} \delta t^{2}\left\|C_{h}^{\mathrm{t}} \epsilon_{h}^{k+1}\right\|_{0}^{2}-\frac{4}{3} \delta t^{2}\left\|C_{h}^{\mathrm{t}} \psi_{h}^{k}\right\|_{0}^{2}-3\left\|\tilde{e}_{h}^{k+1}-e_{h}^{k+1}\right\|_{0}^{2} \\
= & \frac{4}{3} \delta t^{2}\left\|C_{h}^{\mathrm{t}} \epsilon_{h}^{k+1}\right\|_{0}^{2}-\frac{4}{3} \delta t^{2}\left\|C_{h}^{\mathrm{t}} \epsilon_{h}^{k}+\delta_{t} q_{h}^{k+1}\right\|_{0}^{2}-3\left\|\tilde{e}_{h}^{k+1}-e_{h}^{k+1}\right\|_{0}^{2} \\
\geq & \frac{4}{3} \delta t^{2}\left\|C_{h}^{\mathrm{t}} \epsilon_{h}^{k+1}\right\|_{0}^{2}-\frac{4(1+\delta t)}{3} \delta t^{2}\left\|C_{h}^{\mathrm{t}} \epsilon_{h}^{k}\right\|_{0}^{2} \\
& -\frac{4(1+\delta t)}{3 \delta t} \delta t^{2}\left\|C_{h}^{\mathrm{t}} \delta_{t} q_{h}^{k+1}\right\|_{0}^{2}-3\left\|\tilde{e}_{h}^{k+1}-e_{h}^{k+1}\right\|_{0}^{2} \\
\geq & \frac{4}{3} \delta t^{2}\left\|C_{h}^{\mathrm{t}} \epsilon_{h}^{k+1}\right\|_{0}^{2}-\frac{4(1+\delta t)}{3} \delta t^{2}\left\|C_{h}^{\mathrm{t}} \epsilon_{h}^{k}\right\|_{0}^{2}-c \delta t^{3}-3\left\|\tilde{e}_{h}^{k+1}-e_{h}^{k+1}\right\|_{0}^{2} .
\end{aligned}
$$

La constante $c$ est proportionnelle à $\left\|u_{t}\right\|_{L^{\infty}\left(H^{2}\right)}+\left\|p_{t}\right\|_{L^{\infty}\left(H^{1}\right)}$. Noter que c'est à ce niveau seulement qu'une erreur de consistance d'ordre $\delta t$ est introduite. Cette erreur provient du fait que $\psi_{h}^{k}$ n'est pas égal à $\epsilon_{h}^{k}$ mais est égal à $\epsilon_{h}^{k}+\delta_{t} q_{h}^{k+1} ;$ i.e. $\psi_{h}^{k}=\epsilon_{h}^{k}+\mathcal{O}(\delta t)$.

En multipliant (3.10) par $e_{h}^{k+1}$ on obtient

$$
3\left\|e_{h}^{k+1}\right\|_{0}^{2}+3\left\|\tilde{e}_{h}^{k+1}-e_{h}^{k+1}\right\|_{0}^{2}-3\left\|\tilde{e}_{h}^{k+1}\right\|_{0}^{2}=0 .
$$


En sommant toutes ces estimations on obtient

$$
\begin{aligned}
\left\|e_{h}^{k+1}\right\|_{0}^{2}+\left\|2 e_{h}^{k+1}-e_{h}^{k}\right\|_{0}^{2}+ & \frac{4}{3} \delta t^{2}\left\|C_{h}^{\mathrm{t}} \epsilon_{h}^{k+1}\right\|_{0}^{2}+\left\|\delta_{t t} e_{h}^{k+1}\right\|_{0}^{2} \\
+ & 3\left\|\tilde{e}_{h}^{k+1}-e_{h}^{k+1}\right\|_{0}^{2}+(4 \alpha-\gamma) \delta t\left\|\tilde{e}_{h}^{k+1}\right\|_{1}^{2} \\
\leq & \left\|e_{h}^{k}\right\|_{0}^{2}+\left\|2 e_{h}^{k}-e_{h}^{k-1}\right\|_{0}^{2}+\frac{4}{3} \delta t^{2}\left\|C_{h}^{\mathrm{t}} \epsilon_{h}^{k}\right\|_{0}^{2} \\
& \quad+c_{1} \delta t\left(\left\|e_{h}^{k}\right\|_{0}^{2}+\left\|e_{h}^{k-1}\right\|_{0}^{2}+\frac{4}{3} \delta t^{2}\left\|C_{h}^{\mathrm{t}} \epsilon_{h}^{k}\right\|_{0}^{2}\right) \\
& +c_{2} \delta t\left(\left\|\tilde{e}_{h}^{k}-e_{h}^{k}\right\|_{0}^{2}+\left\|\tilde{e}_{h}^{k-1}-e_{h}^{k-1}\right\|_{0}^{2}+\left(\delta t+h^{l+1}\right)^{2}\right) .
\end{aligned}
$$

On choisit $\gamma=3 \alpha$ et on prend $\delta t$ tel que $c_{2} \delta t \leq 1$. En faisant la somme de toutes ces estimations pour $k$ variant de 1 à $n \leq K-1$, on a

$$
\begin{aligned}
\left\|e_{h}^{n+1}\right\|_{0}^{2}+\left\|2 e_{h}^{n+1}-e_{h}^{n}\right\|_{0}^{2}+ & \frac{4}{3} \delta t^{2}\left\|C_{h}^{\mathrm{t}} \epsilon_{h}^{n+1}\right\|_{0}^{2} \\
+ & \sum_{k=1}^{n}\left(\left\|\delta_{t t} e_{h}^{k+1}\right\|_{0}^{2}+\left\|e_{h}^{k+1}-e_{h}^{k+1}\right\|_{0}^{2}\right)+\alpha \delta t \sum_{k=1}^{n}\left\|e_{h}^{k+1}\right\|_{1}^{2} \\
\leq & c_{1}\left(\left\|e_{h}^{1}\right\|_{0}^{2}+\left\|e_{h}^{0}\right\|_{0}^{2}+\delta t^{2}\left\|C_{h}^{\mathrm{t}} \epsilon_{h}^{1}\right\|_{0}^{2}\right) \\
& \quad+c_{2}\left(\delta t+h^{l+1}\right)^{2}+c_{3} \delta t \sum_{k=1}^{n}\left(\left\|e_{h}^{k}\right\|_{0}^{2}+\frac{4}{3} \delta t^{2}\left\|C_{h}^{\mathrm{t}} \epsilon_{h}^{k}\right\|_{0}^{2}\right) .
\end{aligned}
$$

En utilisant l'hypothèse (H1) et le lemme de Gronwall discret on déduit

$$
\|e\|_{l^{\infty}\left(\mathrm{L}^{2}(\Omega)^{d}\right)}+\|\tilde{e}\|_{l^{\infty}\left(\mathrm{L}^{2}(\Omega)^{d}\right)}+\|\tilde{e}\|_{l^{2}\left(\mathrm{H}^{1}(\Omega)^{d}\right)} \leq c\left(\delta t+h^{l+1}\right) .
$$

Les majorations finales résultent des définitions :

$$
\begin{aligned}
& u^{k}-u_{h}^{k}=\eta^{k}+\mathrm{e}_{h}^{k}, \\
& u^{k}-\tilde{u}_{h}^{k}=\eta^{k}+\tilde{\mathrm{e}}_{h}^{k} .
\end{aligned}
$$

C'est à ce niveau seulement que s'introduit l'erreur d'interpolation en $\mathcal{O}\left(h^{l}\right)$ sur l'erreur mesurée en norme $\mathrm{H}^{1}$.

\subsection{Estimations d'ordre deux sur les incréments}

Pour aller vers l'ordre deux il faut obtenir des estimations sur l'incrément des erreurs : $e_{h}^{k+1}-e_{h}^{k}$ et $\tilde{e}_{h}^{k+1}-\tilde{e}_{h}^{k}$. On fait maintenant les hypothèses suivantes :

(H3) $\max \left(\left\|e_{h}^{0}\right\|_{0},\left\|e_{h}^{1}\right\|_{0}\right) \leq c \min \left(h^{l+1}, \delta t h^{l}\right)$, $\max \left(\left\|e_{h}^{0}\right\|_{1},\left\|e_{h}^{1}\right\|_{1}\right) \leq c \delta t^{1 / 2} h^{l}$, $\left\|\epsilon_{h}^{1}\right\|_{1} \leq c h^{l}$.

(H4) $u$ est dans $W^{2, \infty}\left(\mathrm{H}_{0}^{1}(\Omega)^{d} \cap H^{l+1}(\Omega)^{d}\right) \cap H^{3}\left(\mathrm{H}^{1}(\Omega)^{d}\right)$, $p$ est dans $W^{2, \infty}\left(H^{l}(\Omega)\right) \cap H^{3}\left(L^{2}(\Omega)\right)$.

En procédant comme dans [15] : c'est-à-dire, en construisant les équations qui contrôlent les erreurs incrémen- 
tales

$\delta_{t} e^{k+1}, \delta_{t} \tilde{e}^{k+1}$ et $\delta_{t} \epsilon^{k+1}$ et en répétant les arguments de la démonstration du théorème 3.1 on démontre :

Lemme 3.3. Dans le cadre des hypothèses (H3-H4), il existe $c_{s}>0$ et $h_{s}>0$, tel que pour $h$ dans $\left.] 0, h_{s}\right]$ et $\delta t \leq c_{s} /\left(1+\left|\log h^{-1}\right|\right)^{1 / 2}$ en dimension 2 ou $\delta t \leq c_{s} h^{\frac{1}{2}}$ en dimension 3, la solution de l'algorithme de projection (2.11)-(2.12) satisfait les estimations :

$$
\begin{aligned}
\left\|\delta_{t} e_{h}\right\|_{1^{\infty}\left(\mathrm{L}^{2}(\Omega)^{d}\right)} & +\left\|\delta_{t} \tilde{e}_{h}\right\|_{1^{\infty}\left(\mathrm{L}^{2}(\Omega)^{d}\right)}+\delta t\left\|C_{h}^{\mathrm{t}} \delta_{t} \epsilon_{h}\right\|_{1^{\infty}\left(\mathrm{L}^{2}(\Omega)\right)}+\left\|\tilde{e}_{h}-e_{h}\right\|_{1^{\infty}\left(\mathrm{L}^{2}(\Omega)^{d}\right)} \\
& +\left\|\delta_{t} \tilde{e}_{h}\right\|_{1^{2}\left(\mathrm{H}^{1}(\Omega)^{d}\right)}+\delta t^{-\frac{1}{2}}\left\|\delta_{t} e_{h}-\delta_{t} \tilde{e}_{h}\right\|_{1^{2}\left(\mathrm{~L}^{2}(\Omega)^{d}\right)} \leq c \delta t\left(h^{l}+\delta t\right) .
\end{aligned}
$$

La restriction sur le pas de temps résulte de l'inégalité inverse (2.5) qui est utilisée pour contrôler les incréments des résidus non linéaires ; cf. [16] ou [15] pour d'autres détails sur ce type de majoration.

À l'aide de ce lemme on déduit :

Théorème 3.2. Dans le cadre des hypothèses (H3-H4) et si $\delta t$ et $h$ vérifient les restrictions du lemme 3.3, la solution de l'algorithme de projection (2.11)-(2.12) satisfait les estimations :

$$
\left\|u-\tilde{u}_{h}\right\|_{1^{\infty}\left(\mathrm{H}^{1}(\Omega)^{d}\right)}+\left\|p-p_{h}\right\|_{1^{\infty}\left(\mathrm{L}^{2}(\Omega)\right)} \leq c\left(\delta t+h^{l}\right) .
$$

Preuve. On procède en trois étapes :

Étape 1. On reconstruit une équation de quantité de mouvement pour contrôler la pression grâce à la condition "inf-sup." En combinant (3.8)-(2.11) $-i_{h}^{\mathrm{t}}(2.12)$, on obtient

$$
B_{h}^{\mathrm{t}} \epsilon_{h}^{k+1}=-\frac{3 i_{h}^{\mathrm{t}} e_{h}^{k+1}-4 i_{h}^{\mathrm{t}} e_{h}^{k}+i_{h}^{\mathrm{t}} e_{h}^{k-1}}{2 \delta t}+A_{h} \tilde{e}_{h}^{k+1}+\pi_{h}\left[R_{0}^{k+1}+R_{n l}^{k+1}\right] .
$$

La condition "inf-sup" donne

$$
c_{1}\left\|\epsilon_{h}^{k+1}\right\|_{0} \leq \frac{3\left\|\delta_{t} e_{h}^{k+1}\right\|_{0}}{2 \delta t}+\frac{\left\|\delta_{t} e_{h}^{k}\right\|_{0}}{2 \delta t}+c_{2}\left\|\tilde{e}_{h}^{k+1}\right\|_{1}+\sup _{v_{h} \in X_{h},\left\|v_{h}\right\|_{1}=1}\left\langle R_{0}^{k+1}+R_{n l}^{k+1}, v_{h}\right\rangle .
$$

Le contrôle du résidu est classique ( $c f$. [16] pour d'autres détails) : si $\delta t$ et $h$ satisfont les conditions du lemme 3.3, on a l'inégalité suivante

$$
\sup _{v_{h} \in X_{h},\left\|v_{h}\right\|_{1}=1}\left\langle R_{0}^{k+1}+R_{n l}^{k+1}, v_{h}\right\rangle \leq c\left(\delta t^{2}+h^{l+1}+\left\|\tilde{e}_{h}^{k+1}\right\|_{1}+\left\|\tilde{e}_{h}^{k}\right\|_{0}+\left\|\tilde{e}_{h}^{k-1}\right\|_{0}\right) .
$$

Grâce au lemme 3.3 on tire finalement la majoration :

$$
\left\|\epsilon_{h}\right\|_{l^{2}\left(\mathrm{~L}^{2}(\Omega)\right)} \leq c\left(\delta t+h^{l}\right)
$$

Étape 2. En faisant le produit scalaire de (3.13) par $2 \delta_{t} \tilde{e}_{h}^{k+1}$ on obtient

$$
\begin{aligned}
\left\|\nabla \tilde{e}_{h}^{k+1}\right\|_{0}^{2}+\frac{5}{2 \delta t}\left\|\delta_{t} e_{h}^{k+1}\right\|_{0}^{2} \leq & \left\|\nabla \tilde{e}_{h}^{k}\right\|_{0}^{2}+\frac{1}{2 \delta t}\left\|\delta_{t} e_{h}^{k}\right\|_{0}^{2}+\delta t\left\|\epsilon_{h}^{k+1}\right\|_{0}^{2}+\frac{1}{\delta t}\left\|\delta_{t} \tilde{e}_{h}^{k+1}\right\|_{1}^{2} \\
& +\left\langle R_{0}^{k+1}+R_{n l}^{k+1}, 2 \delta_{t} \tilde{e}_{h}^{k+1}\right\rangle \\
\leq & \left\|\nabla \tilde{e}_{h}^{k}\right\|_{0}^{2}+\frac{1}{2 \delta t}\left\|\delta_{t} e_{h}^{k}\right\|_{0}^{2}+\delta t\left\|\epsilon_{h}^{k+1}\right\|_{0}^{2}+\frac{2}{\delta t}\left\|\delta_{t} \tilde{e}_{h}^{k+1}\right\|_{1}^{2} \\
& +\sup _{v_{h} \in X_{h},\left\|v_{h}\right\|_{1}=1} \delta t\left\langle R_{0}^{k+1}+R_{n l}^{k+1}, v_{h}\right\rangle^{2} .
\end{aligned}
$$


De cette majoration on tire (grâce au lemme de Gronwall discret, au lemme 3.3 et à la majoration sur $\left\|\epsilon_{h}\right\|_{l^{2}\left(\mathrm{~L}^{2}(\Omega)\right)}$ obtenue ci-dessus) l'estimation :

$$
\left\|\tilde{e}_{h}\right\|_{l^{\infty}\left(\mathrm{H}^{1}(\Omega)^{d}\right)} \leq c\left(\delta t+h^{l}\right) .
$$

Étape 3. Les estimations désirées résultent des étapes 1 et 2 et des définitions :

$$
u\left(t^{k}\right)-\tilde{u}_{h}^{k}=\eta^{k}+\tilde{e}_{h}^{k}, \quad p\left(t^{k}\right)-p_{h}^{k}=\mu^{k}+\epsilon_{h}^{k} .
$$

La démonstration du théorème est complète.

\section{Estimations D'ORDRE DEUX}

Avant de démontrer l'ordre deux, on établit quelques résultats préliminaires. En particulier on introduit l'approximation de l'inverse à droite de l'opérateur de Stokes.

\subsection{L'inverse à droite discret de l'opérateur de Stokes}

On définit l'opérateur $S_{h}: H_{h} \longrightarrow H_{h}$ tel que pour tout $v_{h}$ dans $H_{h}$, le couple $\left(S_{h} v_{h}, r_{h}\right) \in X_{h} \times M_{h}$ soit la solution du problème de Stokes discret dual :

$$
\left\{\begin{array}{l}
A_{h}^{\mathrm{t}}\left(S_{h} v_{h}\right)+B_{h}^{\mathrm{t}} r_{h}=i_{h}^{\mathrm{t}} v_{h} \\
B_{h} S_{h} v_{h}=0
\end{array}\right.
$$

Cette définition a bien un sens car $S_{h} v_{h} \in \operatorname{ker}\left(B_{h}\right) \subset \operatorname{ker}\left(C_{h}\right)=H_{h}$, puisque $C_{h}$ est un prolongement de $B_{h}$. L'opérateur $S_{h}$ est l'équivalent discret de l'inverse à droite de l'opérateur de Stokes. Il a les propriétés suivantes.

Lemme 4.1. La forme bilinéaire $\left(v_{h}, w_{h}\right) \longmapsto\left(v_{h}, S_{h} w_{h}\right)$ agissant sur $H_{h} \times H_{h}$ est symétrique positive et sa restriction à $V_{h} \times V_{h}$ est définie.

Preuve. Soit $\left(v_{h}, w_{h}\right)$ dans $H_{h} \times H_{h}$. De l'identité $\left(v_{h}, S_{h} w_{h}\right)=\left(A_{h}^{\mathrm{t}} S_{h} v_{h}, S_{h} w_{h}\right)$ on déduit que la forme bilinéaire est symétrique et positive (car $A_{h}$ est auto-adjoint et positif). Supposons que $v_{h}$ est dans $V_{h}$, grâce à la coercivité de $A_{h}$ on déduit que $\left(v_{h}, S_{h} v_{h}\right)$ est nul si et seulement si $S_{h} v_{h}$ est nul ; c'est-à-dire, en revenant à la définition de $S_{h} v_{h}, i_{h}^{\mathrm{t}} i_{h} v_{h}=v_{h}$ est dans l'image de $B_{h}^{\mathrm{t}}$. En conclusion $v_{h}$ appartient à $\operatorname{ker}\left(B_{h}\right) \cap \operatorname{im}\left(B_{h}^{\mathrm{t}}\right)$, c'est-à-dire $v_{h}=0$.

Remarque 4.1. La forme linéaire $v_{h} \longmapsto\left(v_{h}, S_{h} v_{h}\right)^{1 / 2}$ est une norme sur $V_{h}$. Par la suite on note (abusivement) $\left\|v_{h}\right\|_{*}$ la quantité $\left(v_{h}, S_{h} v_{h}\right)^{1 / 2}$ pour tout $v_{h}$ dans $H_{h}$.

Lemme 4.2. Il existe $c>0$ tel que pour tout $v_{h}$ dans $H_{h}$ on $a$ :

$$
\left\|S_{h} v_{h}\right\|_{1} \leq c\left\|v_{h}\right\|_{*} .
$$

Preuve. Il suffit de multiplier (4.1) par $S_{h} v_{h}$, puis d'utiliser l'inégalité de Poincaré et la définition de $\left\|v_{h}\right\|_{*}$.

Lemme 4.3. Il existe $c>0$ tel que pour tout $v_{h}$ dans $H_{h}$ on $a:$

$$
\left\|A_{h}^{\mathrm{t}} S_{h} v_{h}\right\|_{0} \leq c\left\|v_{h}\right\|_{0}, \text { et } \quad\left\|C_{h}^{\mathrm{t}} r_{h}\right\|_{0} \leq c\left\|v_{h}\right\|_{0} .
$$


Preuve. Soit $(s, r) \in \mathrm{H}_{0}^{1}(\Omega)^{d} \times \mathrm{L}_{0}^{2}(\Omega)$ la solution du problème de Stokes dual

$$
\begin{cases}(\nabla w, \nabla s)-(r, \nabla \cdot w)=\left(v_{h}, w\right), & \forall w \in \mathrm{H}_{0}^{1}(\Omega)^{d}, \\ (q, \nabla \cdot s)=0, & \forall q \in \mathrm{L}_{0}^{2}(\Omega) .\end{cases}
$$

D'après les propriétés régularisantes de l'opérateur de Stokes $(c f .[1,6])$, on a

$$
\|s\|_{2} \leq c\left\|v_{h}\right\|_{0} \text {, et } \quad\|r\|_{1} \leq c\left\|v_{h}\right\|_{0} .
$$

De la théorie de l'approximation du problème de Stokes on déduit :

$$
\left\|s-S_{h} v_{h}\right\|_{1}+\left\|r-r_{h}\right\|_{0} \leq \operatorname{ch}\|s\|_{2} .
$$

En utilisant une inégalité inverse, on a

$$
\begin{aligned}
\left\|A_{h}^{\mathrm{t}} S_{h} v_{h}\right\|_{0} & =\sup _{w_{h} \in X_{h}-\{0\}}\left(A_{h}^{\mathrm{t}} S_{h} v_{h}, w_{h}\right) /\left\|w_{h}\right\|_{0} \\
& =\sup _{w_{h} \in X_{h}-\{0\}}\left(\nabla S_{h} v_{h}, \nabla w_{h}\right) /\left\|w_{h}\right\|_{0} \\
& \leq \sup _{w_{h} \in X_{h}-\{0\}}\left(\nabla\left(S_{h} v_{h}-s\right), \nabla w_{h}\right) /\left\|w_{h}\right\|_{0}+\sup _{w_{h} \in X_{h}-\{0\}}\left(\nabla s, \nabla w_{h}\right) /\left\|w_{h}\right\|_{0} \\
& \leq c h^{-1}\left\|S_{h} v_{h}-s\right\|_{1}+\|\Delta s\|_{0} \\
& \leq c\|s\|_{2} \\
& \leq c\left\|v_{h}\right\|_{0} .
\end{aligned}
$$

On déduit la seconde inégalité en utilisant la stabilité en norme $H^{1}$ de $C_{h}^{\mathrm{t}}$ (cf. proposition 2.1) :

$$
\begin{aligned}
\left\|C_{h}^{\mathrm{t}} r_{h}\right\|_{0} & \leq c\|r\|_{1} \\
& \leq c\left\|v_{h}\right\|_{0} .
\end{aligned}
$$

Ce lemme permet à son tour de montrer le résultat suivant qui sera déterminant par la suite.

Lemme 4.4. Il existe $c>0$ tel que pour tout $v_{h}$ dans $X_{h}$ et tout $w_{h}$ dans $H_{h}$ on a:

$$
\left(A_{h} v_{h}, S_{h} w_{h}\right) \geq\left(P_{H_{h}} i_{h} v_{h}, w_{h}\right)-c\left\|w_{h}\right\|_{0}\left\|i_{h} v_{h}-P_{H_{h}} i_{h} v_{h}\right\|_{0} .
$$

Preuve. On a

$$
\begin{aligned}
\left(A_{h} v_{h}, S_{h} w_{h}\right) & =\left(v_{h}, A_{h}^{\mathrm{t}} S_{h} w_{h}\right) \\
& =\left(v_{h}, i_{h}^{\mathrm{t}} w_{h}\right)-\left(v_{h}, B_{h}^{\mathrm{t}} r_{h}\right) \\
& =\left(i_{h} v_{h}, w_{h}\right)-\left(v_{h}, i_{h}^{\mathrm{t}} C_{h}^{\mathrm{t}} r_{h}\right) \\
& =\left(P_{H_{h}} i_{h} v_{h}, w_{h}\right)-\left(i_{h} v_{h}-P_{H_{h}} i_{h} v_{h}, C_{h}^{\mathrm{t}} r_{h}\right) \\
& \geq\left(P_{H_{h}} i_{h} v_{h}, w_{h}\right)-\left\|i_{h} v_{h}-P_{H_{h}} i_{h} v_{h}\right\|_{0}\left\|C_{h}^{\mathrm{t}} r_{h}\right\|_{0} .
\end{aligned}
$$

On déduit le résultat cherché en utilisant la majoration $\left\|C_{h}^{\mathrm{t}} r_{h}\right\|_{0} \leq c\left\|w_{h}\right\|_{0}$ obtenue au lemme précédent. 
Remarque 4.2. On comprend mieux l'intérêt de cette minoration en posant $w_{h}=P_{H_{h}} i_{h} v_{h}$. Dans ce cas particulier, pour tout $\epsilon \in] 0,1[$ on obtient :

$$
\left(A_{h} v_{h}, S_{h} P_{H_{h}} i_{h} v_{h}\right) \geq(1-\epsilon)\left\|P_{H_{h}} i_{h} v_{h}\right\|_{0}^{2}-c_{\epsilon}\left\|i_{h} v_{h}-P_{H_{h}} i_{h} v_{h}\right\|_{0}^{2} .
$$

Cette inégalité fournit un contrôle sur $\left\|P_{H_{h}} i_{h} v_{h}\right\|_{0}$.

\subsection{L'ordre deux en norme $\mathbf{l}^{2}\left(\mathbf{L}^{2}(\Omega)^{\mathrm{d}}\right)$ sur la vitesse}

Avant de donner le résultat principal de cette section, on présente de façon heuristique les lignes directrices du raisonnement qui permet de démontrer l'ordre deux en temps. On reconstruit tout d'abord une équation de quantité de mouvement pour la quantité $i_{h}^{\mathrm{t}} e_{h}^{k+1}$ en combinant $(3.8)-(2.11)-i_{h}^{\mathrm{t}}(2.12)$.

$$
\frac{3 i_{h}^{\mathrm{t}} e_{h}^{k+1}-4 i_{h}^{\mathrm{t}} e_{h}^{k}+i_{h}^{\mathrm{t}} e_{h}^{k-1}}{2 \delta t}+A_{h} \tilde{e}_{h}^{k+1}+B_{h}^{\mathrm{t}} \epsilon_{h}^{k+1}=\pi_{h}\left[R_{0}^{k+1}+R_{n l}^{k+1}\right]
$$

La linéarisation du terme convectif et l'approximation de la dérivée temporelle sont construites pour être consistantes à l'ordre deux. On peut donc s'attendre à ce que les résidus correspondants soient du second ordre en $\delta t$, d'ailleurs l'étape 2 de la preuve du théorème 3.1 fait clairement apparaître que la seule erreur de consistance d'ordre un en $\delta t$ est introduite par le décalage temporelle sur la pression. Pour éliminer l'erreur de consistance liée à la pression, il suffit de tester l'équation (4.6) par une fonction dans $V_{h}$ (c'est-à-dire utiliser une fonction test à divergence discrète nulle). L'introduction de l'opérateur $S_{h}$ est motivée par le fait que $S_{h} e_{h}^{k+1}$ est un bon candidat pour tester (4.6). En effet, lorsqu'on fait le produit scalaire de $S_{h} e_{h}^{k+1}$ avec (4.6), l'approximation de la dérivée temporelle fait apparaître des normes $\|\cdot\|_{*}$ qui se contrôlent facilement ; le terme de pression disparaît naturellement; les résidus sont a priori contôlables à l'ordre deux ; le terme le plus gênant est $\left(A_{h} \tilde{e}_{h}^{k+1}, S_{h} e_{h}^{k+1}\right)$. Or d'après ce qui précède on a

$$
\left(A_{h} \tilde{e}_{h}^{k+1}, S_{h} e_{h}^{k+1}\right) \geq(1-\epsilon)\left\|e_{h}^{k+1}\right\|_{0}^{2}-c_{\epsilon}\left\|\tilde{e}_{h}^{k+1}-e_{h}^{k+1}\right\|_{0}^{2} .
$$

Ce terme permet donc un contrôle en norme $\mathrm{L}^{2}$ de $e_{h}^{k+1}$ au terme d'erreur près $\left\|\tilde{e}_{h}^{k+1}-e_{h}^{k+1}\right\|_{0}$ qu'il est possible de contrôler en utilisant les estimations d'ordre deux établis pour les incréments des erreurs (cf. lemme 3.3).

Le résultat essentiel de cette section est donc :

Théorème 4.1. Sous les hypothèses (H3-H4) et les conditions sur $\delta t$ et $h$ du lemme 3.3, la solution de l'algorithme (2.11)-(2.12) vérifie:

$$
\left\|u-u_{h}\right\|_{l^{2}\left(\mathrm{~L}^{2}(\Omega)^{d}\right)}+\left\|u-\tilde{u}_{h}\right\|_{l^{2}\left(\mathrm{~L}^{2}(\Omega)^{d}\right)} \leq c\left(\delta t^{2}+h^{l+1}\right)
$$

Preuve. On procède en trois étapes :

Étape 1. On reconstruit l'équation de quantité de mouvement (4.6) qui contrôle $i_{h}^{\mathrm{t}} e_{h}^{k+1}$ en combinant (3.8)-(2.11) $-i_{h}^{\mathrm{t}}(2.12)$.

Étape 2. On multiplie (4.6) par la fonction test : $4 \delta t S_{h} e_{h}^{k+1}$. En utilisant la symétrie de la forme bilinéaire $\left(v_{h}, S_{h} w_{h}\right)$ (cf. lemme 4.1) on obtient :

$$
\begin{aligned}
\left\|e_{h}^{k+1}\right\|_{*}^{2}+\left\|2 e_{h}^{k+1}-e_{h}^{k}\right\|_{*}^{2} & +\left\|\delta_{t t} e_{h}^{k+1}\right\|_{*}^{2}+4 \delta t\left(A_{h} \tilde{e}_{h}^{k+1}, S_{h} e_{h}^{k+1}\right) \\
& \leq\left\|e_{h}^{k}\right\|_{*}^{2}+\left\|2 e_{h}^{k}-e_{h}^{k-1}\right\|_{*}^{2}+4 \delta t\left\langle R_{0}^{k+1}+R_{n l}^{k+1}, S_{h} e_{h}^{k+1}\right\rangle .
\end{aligned}
$$


En remarquant que $e_{h}^{k+1}=P_{H_{h}} i_{h} \tilde{e}_{h}^{k+1}$, on applique le lemme 4.4 avec $\epsilon=1 / 4$ dans l'inéquation (4.5) et on obtient

$$
\begin{aligned}
\left\|e_{h}^{k+1}\right\|_{*}^{2}+\left\|2 e_{h}^{k+1}-e_{h}^{k}\right\|_{*}^{2}+ & \left\|\delta_{t t} e_{h}^{k+1}\right\|_{0}^{2}+3 \delta t\left\|e_{h}^{k+1}\right\|_{0}^{2} \\
\leq & \left\|e_{h}^{k}\right\|_{*}^{2}+\left\|2 e_{h}^{k}-e_{h}^{k-1}\right\|_{*}^{2}+c \delta t\left\|e_{h}^{k+1}-i_{h} \tilde{e}_{h}^{k+1}\right\|_{0}^{2} \\
& +4 \delta t\left\langle R_{0}^{k+1}+R_{n l}^{k+1}, S_{h} e_{h}^{k+1}\right\rangle .
\end{aligned}
$$

On contrôle le premier reste en utilisant la majoration du lemme 4.2 :

$$
\begin{aligned}
4 \delta t\left\langle R_{0}^{k+1}, S_{h} e_{h}^{k+1}\right\rangle \leq & 4 \delta t\left\|R_{0}^{k+1}\right\|_{-1}\left\|S_{h} e_{h}^{k+1}\right\|_{1} \\
\leq & \delta t\left\|e_{h}^{k+1}\right\|_{*}^{2}+c \delta t\left\|R_{0}^{k+1}\right\|_{-1}^{2} \\
\leq & \quad \delta t\left\|e_{h}^{k+1}\right\|_{*}^{2}+c \delta t\left[h^{l+1}\left(\left\|u_{t}\right\|_{L^{\infty}\left(H^{l+1}\right)}+\left\|p_{t}\right\|_{L^{\infty}\left(H^{l}\right)}\right)\right. \\
& \left.\quad+\delta t^{2}\left(\left\|u_{t t t}\right\|_{L^{\infty}\left(H^{1}\right)}+\left\|p_{t t t}\right\|_{L^{\infty}\left(L^{2}\right)}\right)\right]^{2} \\
& \leq \delta t\left\|\tilde{e}_{h}^{k+1}\right\|_{*}^{2}+c \delta t\left(h^{l+1}+\delta t^{2}\right)^{2} .
\end{aligned}
$$

Maintenant on contrôle le reste induit par les termes non linéaires. On décompose ce reste comme suit :

$$
\begin{aligned}
-\left\langle R_{n l}^{k+1}, S_{h} e_{h}^{k+1}\right\rangle= & d\left(\delta_{t t} u^{k+1}, u^{k+1}, S_{h} e_{h}^{k+1}\right)+d\left(2 \eta^{k}-\eta^{k-1}, u^{k+1}, S_{h} e_{h}^{k+1}\right) \\
& +d\left(2 w_{h}^{k}-w_{h}^{k-1}, \eta^{k+1}, S_{h} e_{h}^{k+1}\right)+d\left(2 \tilde{e}_{h}^{k}-\tilde{e}_{h}^{k-1}, w_{h}^{k+1}, S_{h} e_{h}^{k+1}\right) \\
& +d\left(2 w_{h}^{k}-w_{h}^{k-1}, \tilde{e}_{h}^{k+1}, S_{h} e_{h}^{k+1}\right)-d\left(2 \tilde{e}_{h}^{k}-\tilde{e}_{h}^{k-1}, \tilde{e}_{h}^{k+1}, S_{h} e_{h}^{k+1}\right) .
\end{aligned}
$$

On note $R_{1}\left(S_{h} e_{h}^{k+1}\right), \ldots, R_{6}\left(S_{h} e_{h}^{k+1}\right)$ les six restes. On donne maintenant une majoration pour chacun de ces termes. En utilisant la majoration du lemme 4.2 on a

$$
\begin{gathered}
4 \delta t\left|R_{1}\left(S_{h} e_{h}^{k+1}\right)\right| \leq c \delta t\left\|\delta_{t t} u^{k+1}\right\|_{0}\left\|u^{k+1}\right\|_{2}\left\|S_{h} e_{h}^{k+1}\right\|_{1} \\
\leq c \delta t^{3}\left\|u_{t t}\right\|_{L^{\infty}\left(L^{2}\right)}\|u\|_{L^{\infty}\left(H^{2}\right)}\left\|e_{h}^{k+1}\right\|_{*} \\
\leq c \delta t^{5}+\delta t\left\|e_{h}^{k+1}\right\|_{*}^{2} . \\
4 \delta t\left|R_{2}\left(S_{h} e_{h}^{k+1}\right)\right| \leq c \delta t\left\|2 \eta^{k}-\eta^{k-1}\right\|_{0}\left\|u^{k+1}\right\|_{2}\left\|S_{h} e_{h}^{k+1}\right\|_{1} \\
\leq c \delta t h^{l+1}\left(\|u\|_{L^{\infty}\left(H^{l+1}\right)}+\|p\|_{L^{\infty}\left(H^{l}\right)}\right)\left\|e_{h}^{k+1}\right\|_{*} \\
\leq c \delta t h^{2(l+1)}+\delta t\left\|e_{h}^{k+1}\right\|_{*}^{2} . \\
4 \delta t\left|R_{3}\left(S_{h} e_{h}^{k+1}\right)\right| \leq c \delta t\left(\left\|2 w_{h}^{k}-w_{h}^{k-1}\right\|_{1,3}+\left\|2 w_{h}^{k}-w_{h}^{k-1}\right\|_{0, \infty}\right)\left\|\eta^{k+1}\right\|_{0,2}\left\|S_{h} e_{h}^{k+1}\right\|_{1,2} \\
\leq c \delta t h^{l+1}\left(\|u\|_{L^{\infty}\left(H^{l+1}\right)}+\|p\|_{L^{\infty}\left(H^{l}\right)}\right)\left\|S_{h} e_{h}^{k+1}\right\|_{1,2} \\
\leq c \delta t h^{2(l+1)}+\delta t\left\|e_{h}^{k+1}\right\|_{*}^{2} .
\end{gathered}
$$


En utilisant l'inégalité $\left\|\tilde{e}_{h}^{k+1}\right\|_{0} \leq\left\|\tilde{e}_{h}^{k+1}-e_{h}^{k}\right\|_{0}+\left\|e_{h}^{k}\right\|_{0}$, on déduit

$$
\begin{aligned}
4 \delta t\left|R_{4}\left(S_{h} e_{h}^{k+1}\right)\right| & \leq c \delta t\left(2\left\|\tilde{e}_{h}^{k}\right\|_{0,2}+\left\|\tilde{e}_{h}^{k-1}\right\|_{0,2}\right)\left(\left\|w_{h}^{k}\right\|_{1,3}+\left\|w_{h}^{k}\right\|_{0, \infty}\right)\left\|S_{h} e_{h}^{k+1}\right\|_{1,2} \\
& \leq c \delta t\left(2\left\|\tilde{e}_{h}^{k}\right\|_{0,2}+\left\|\tilde{e}_{h}^{k-1}\right\|_{0,2}\right)\left(\|u\|_{L^{\infty}\left(H^{2}\right)}+\|p\|_{L^{\infty}\left(H^{1}\right)}\right)\left\|e_{h}^{k+1}\right\|_{*} \\
& \leq c \delta t\left(\left\|\tilde{e}_{h}^{k}-e_{h}^{k}\right\|_{0}+\left\|\tilde{e}_{h}^{k-1}-e_{h}^{k-1}\right\|_{0}+\left\|\delta_{t} e_{h}^{k}\right\|_{0}+\left\|e_{h}^{k}\right\|_{0}\right)\left\|e_{h}^{k+1}\right\|_{*} .
\end{aligned}
$$

Maintenant on utilise le résultat du lemme 3.3 pour borner $\left\|\tilde{e}_{h}^{k}-e_{h}^{k}\right\|_{0}+\left\|\tilde{e}_{h}^{k-1}-e_{h}^{k-1}\right\|_{0}+\left\|\delta_{t} e_{h}^{k}\right\|_{0}$ par $c \delta t\left(\delta t+h^{l}\right)$. En remarquant que pour $l \geq 1$ et $h \leq 1$, on a $2 \delta t h^{l} \leq \delta t^{2}+h^{l+1}$, on peut majorer $c \delta t\left(\delta t+h^{l}\right)$ par $c\left(\delta t^{2}+h^{l+1}\right)$. Finalement, on obtient

$$
4 \delta t\left|R_{4}\left(S_{h} e_{h}^{k+1}\right)\right| \leq \gamma \delta t\left\|e_{h}^{k}\right\|_{0}^{2}+c_{1} \delta t\left\|e_{h}^{k+1}\right\|_{*}^{2}+c_{2} \delta t\left(\delta t^{2}+h^{l+1}\right)^{2}
$$

On procède de la même façon pour le cinquième terme :

$$
\begin{aligned}
4 \delta t\left|R_{5}\left(S_{h} e_{h}^{k+1}\right)\right| & \leq c \delta t\left(\left\|2 w_{h}^{k}-w_{h}^{k-1}\right\|_{1,3}+\left\|2 w_{h}^{k}-w_{h}^{k-1}\right\|_{0, \infty}\right)\left\|\tilde{e}_{h}^{k+1}\right\|_{0,2}\left\|S_{h} e_{h}^{k+1}\right\|_{1,2} \\
& \leq c \delta t\left(\|u\|_{L^{\infty}\left(H^{2}\right)}+\|p\|_{L^{\infty}\left(H^{1}\right)}\right)\left\|\tilde{e}_{h}^{k+1}\right\|_{0,2}\left\|e_{h}^{k+1}\right\|_{*} \\
& \leq c \delta t\left(\left\|\tilde{e}_{h}^{k+1}-e_{h}^{k+1}\right\|_{0,2}+\left\|e_{h}^{k+1}\right\|_{0,2}\right)\left\|e_{h}^{k+1}\right\|_{*} \\
& \leq \gamma \delta t\left\|e_{h}^{k+1}\right\|_{0}^{2}+c_{1} \delta t\left\|e_{h}^{k+1}\right\|_{*}^{2}+c_{2} \delta t\left(\delta t^{2}+h^{l+1}\right)^{2} .
\end{aligned}
$$

Pour le dernier terme on a :

$$
4 \delta t\left|R_{6}\left(S_{h} e_{h}^{k+1}\right)\right| \leq c \delta t\left\|2 \tilde{e}_{h}^{k}-\tilde{e}_{h}^{k-1}\right\|_{1}\left\|\tilde{e}_{h}^{k+1}\right\|_{1}\left\|S_{h} e_{h}^{k+1}\right\|_{1} .
$$

On utilise le résultat du théorème 3.2 pour borner $\left\|\tilde{e}_{h}^{k-1}\right\|_{1},\left\|\tilde{e}_{h}^{k}\right\|_{1}$ et $\left\|\tilde{e}_{h}^{k+1}\right\|_{1}$ par $c\left(\delta t+h^{l}\right)$. En remarquant que $h^{4 l} \leq h^{2(l+1)}$ dès que $l \geq 1$ et $h \leq 1$, on obtient finalement :

$$
4 \delta t\left|R_{6}\left(S_{h} e_{h}^{k+1}\right)\right| \leq c \delta t\left(\delta t^{2}+h^{l+1}\right)^{2}+\delta t\left\|e_{h}^{k+1}\right\|_{*}^{2} .
$$

En combinant toutes les majorations obtenues on déduit l'inégalité suivante :

$$
\begin{aligned}
\left\|e_{h}^{k+1}\right\|_{*}^{2}+\left\|2 e_{h}^{k+1}-e_{h}^{k}\right\|_{*}^{2}+ & \left\|\delta_{t t} e_{h}^{k+1}\right\|_{0}^{2}+3 \delta t\left\|e_{h}^{k+1}\right\|_{0}^{2} \\
\leq & \left\|e_{h}^{k}\right\|_{*}^{2}+\left\|2 e_{h}^{k}-e_{h}^{k-1}\right\|_{*}^{2}+c \delta t\left(\delta t^{2}+h^{l+1}\right)^{2} \\
& +\gamma \delta t\left\|e_{h}^{k+1}\right\|_{0}^{2}+\gamma \delta t\left\|e_{h}^{k}\right\|_{0}^{2}+c \delta t\left\|e_{h}^{k+1}\right\|_{*}^{2} .
\end{aligned}
$$

Étape 3. On choisit $\gamma \leq 1$. En sommant de $k=1$ à $n \leq K-1$ on obtient

$$
\begin{array}{r}
\left\|e_{h}^{n+1}\right\|_{*}^{2}+\left\|2 e_{h}^{n+1}-e_{h}^{n}\right\|_{*}^{2}+ \\
\leq \sum_{k=1}^{n}\left\|\delta_{t t} e_{h}^{n+1}\right\|_{0}^{2}+\delta t \sum_{k=1}^{n}\left\|e_{h}^{k+1}\right\|_{0}^{2} \\
\leq\left\|e_{h}^{1}\right\|_{*}^{2}+\left\|2 e_{h}^{1}-e_{h}^{0}\right\|_{*}^{2}+\delta t\left\|e_{h}^{1}\right\|_{0}^{2} \\
+c\left(\delta t^{2}+h^{l+1}\right)^{2}+c \delta t \sum_{k=1}^{n+1}\left\|e_{h}^{k}\right\|_{*}^{2} .
\end{array}
$$

En appliquant le lemme de Gronwall discret on obtient :

$$
\left\|e_{h}\right\|_{l^{\infty}\left(\|\cdot\|_{*}\right)}+\left\|e_{h}\right\|_{l^{2}\left(\mathrm{~L}^{2}(\Omega)^{d}\right)} \leq c\left(\delta t^{2}+h^{l+1}\right) .
$$

Le résultat final découle de cette majoration, du lemme 3.3 et des définitions de $e_{h}$ et $\tilde{e}_{h}$. 


\subsection{Estimation en norme $\mathbf{1}^{\infty}\left(\mathbf{L}^{2}(\Omega)^{\mathrm{d}}\right)$ de l'erreur sur la vitesse}

On cherche maintenant à obtenir une estimation de l'erreur en norme $l^{\infty}\left(\mathrm{L}^{2}(\Omega)^{d}\right)$. Dans un premier temps on cherche un contrôle de l'erreur incrémentale $\delta_{t} e_{h}^{k+1}$ et on fait les hypothèses suivantes

(H5) $\max \left(\left\|e_{h}^{0}\right\|_{0},\left\|e_{h}^{1}\right\|_{0}\right) \leq c \min \left(h^{l+1}, \delta t^{3 / 2} h^{l}\right)$.

(H6) $u \in W^{3, \infty}\left(H^{l+1}(\Omega)^{d} \cap \mathrm{H}_{0}^{1}(\Omega)^{d}\right) \cap W^{4, \infty}\left(\mathrm{H}^{1}(\Omega)^{d}\right)$ et $p \in W^{3, \infty}\left(H^{l}(\Omega)\right) \cap W^{4, \infty} L\left(\mathrm{~L}^{2}(\Omega)\right)$.

On donne d'abord deux lemmes qui permettent de contrôler les résidus linaires et non linéaires. En procédant comme dans la démonstration du théorème 4.1 on déduit

Lemme 4.5. Sous les hypothèses du théorème 3.2, on a:

$$
\sup _{v_{h} \in X_{h},\left\|v_{h}\right\|_{1}=1}\left\langle R_{0}^{k+1}+R_{n l}^{k+1}, v_{h}\right\rangle \leq c\left(\delta t^{2}+h^{l+1}+\left\|e_{h}^{k+1}\right\|_{0}+\left\|e_{h}^{k}\right\|_{0}+\left\|e_{h}^{k-1}\right\|_{0}\right) .
$$

Le contrôle du résidu incrémental est fourni par :

Lemme 4.6. Sous les hypothèses du théorème 3.2 et (H6), on a :

$$
\begin{aligned}
\sup _{v_{h} \in X_{h},\left\|v_{h}\right\|_{1}=1}\left\langle\delta_{t} R_{0}^{k+1}\right. & \left.+\delta_{t} R_{n l}^{k+1}, v_{h}\right\rangle \leq c_{1} \delta t\left(\delta t^{2}+h^{l+1}\right) \\
& +c_{2} \delta t\left(\left\|e_{h}^{k+1}\right\|_{0}+\left\|e_{h}^{k}\right\|_{0}+\left\|e_{h}^{k-1}\right\|_{0}\right) \\
& +c_{3}\left(\left\|\delta_{t} e_{h}^{k+1}\right\|_{0}+\left\|\delta_{t} e_{h}^{k}\right\|_{0}+\left\|\delta_{t} e_{h}^{k-1}\right\|_{0}\right) \\
& +c_{4} \sum_{l=k-1}^{k+1}\left\|\delta_{t}\left(\tilde{e}_{h}^{l}-e_{h}^{l}\right)\right\|_{0} .
\end{aligned}
$$

On établit maintenant une majoration en $\mathcal{O}\left(\delta t^{5 / 2}\right)$ sur l'incrément $\delta_{t} e_{h}^{k+1}$.

Lemme 4.7. Sous les hypothèses du théorème 3.2 et (H5-H6), on a :

$$
\left\|\delta_{t} e\right\|_{l^{2}\left(\mathrm{~L}^{2}(\Omega)^{d}\right)} \leq c_{1} \delta t^{3 / 2}\left(\delta t+h^{l}\right)+c_{2} \delta t\left(\delta t^{2}+h^{l+1}\right) .
$$

Preuve. On procède en trois étapes.

Étape 1. Les erreurs incrémentales sont contrôlées par l'équation

$$
\frac{3 i_{h}^{\mathrm{t}} \delta_{t} e_{h}^{k+1}-4 i_{h}^{\mathrm{t}} \delta_{t} e_{h}^{k}+i_{h}^{\mathrm{t}} \delta_{t} e_{h}^{k-1}}{2 \delta t}+A_{h} \delta_{t} \tilde{e}_{h}^{k+1}+B_{h}^{\mathrm{t}} \delta_{t} \epsilon_{h}^{k+1}=\pi_{h}\left[\delta_{t} R_{0}^{k+1}+\delta_{t} R_{n l}^{k+1}\right] .
$$


Étape 2. On multiplie (4.11) par la fonction test $4 \delta t S_{h} \delta_{t} e_{h}^{k+1}$, et en appliquant le lemme 4.4 (avec $\epsilon=1 / 4$ dans l'inéquation (4.5)) et on obtient

$$
\begin{aligned}
\left\|\delta_{t} e_{h}^{k+1}\right\|_{*}^{2}+\| 2 \delta_{t} e_{h}^{k+1}- & \delta_{t} e_{h}^{k}\left\|_{*}^{2}+\right\| \delta_{t t} e_{h}^{k+1}\left\|_{0}^{2}+3 \delta t\right\| \delta_{t} e_{h}^{k+1} \|_{0}^{2} \\
\leq & \left\|\delta_{t} e_{h}^{k}\right\|_{*}^{2}+\left\|2 \delta_{t} e_{h}^{k}-\delta_{t} e_{h}^{k-1}\right\|_{*}^{2}+c \delta t\left\|\delta_{t} e_{h}^{k+1}-i_{h} \delta_{t} \tilde{e}_{h}^{k+1}\right\|_{0}^{2} \\
& \quad+4 \delta t\left\langle\delta_{t} R_{0}^{k+1}+\delta_{t} R_{n l}^{k+1}, S_{h} \delta_{t} e_{h}^{k+1}\right\rangle, \\
\leq & \left\|\delta_{t} e_{h}^{k}\right\|_{*}^{2}+\left\|2 \delta_{t} e_{h}^{k}-\delta_{t} e_{h}^{k-1}\right\|_{*}^{2}+c \delta t\left\|\delta_{t} e_{h}^{k+1}-i_{h} \delta_{t} \tilde{e}_{h}^{k+1}\right\|_{0}^{2} \\
& \quad+c_{\gamma} \delta t\left\|\delta_{t} e_{h}^{k+1}\right\|_{*}^{2}+\gamma \delta t \sup _{v_{h} \in X_{h},\left\|v_{h}\right\|_{1}=1}\left\langle\delta_{t} R_{0}^{k+1}+\delta_{t} R_{n l}^{k+1}, v_{h}\right\rangle^{2},
\end{aligned}
$$

où $\gamma>0$ peut être choisi suffisamment petit.

Étape 3. En utilisant la majoration du residu fournie par le lemme 4.6, en utilisant les majorations du lemme (3.3), en choisissant $\gamma$ suffisamment petit et en sommant de $k=2$ à $n \leq K-1$, on obtient

$$
\begin{aligned}
\left\|\delta_{t} e_{h}^{n+1}\right\|_{*}^{2}+\left\|2 \delta_{t} e_{h}^{n+1}-\delta_{t} e_{h}^{n}\right\|_{*}^{2}+ & \sum_{k=2}^{n}\left\|\delta_{t t} e_{h}^{k+1}\right\|_{0}^{2}+\delta t \sum_{k=2}^{n}\left\|\delta_{t} e_{h}^{k+1}\right\|_{0}^{2} \\
\leq & c_{1}\left\|\delta_{t} e_{h}^{2}\right\|_{*}^{2}+c_{2}\left\|\delta_{t} e_{h}^{1}\right\|_{*}^{2}+c_{3} \delta t \sum_{k=2}^{n}\left\|\delta_{t} e_{h}^{k+1}\right\|_{*}^{2} \\
& +c_{4} \delta t^{2}\left(\delta t^{2}+h^{l+1}\right)^{2}+c_{5} \delta t^{3}\left(\delta t+h^{l}\right)^{2} .
\end{aligned}
$$

L'hypothèse d'initialisation (H5) implique :

$$
\max \left(\left\|\delta_{t} e_{h}^{2}\right\|_{*},\left\|\delta_{t} e_{h}^{1}\right\|_{*}\right) \leq c_{1} \delta t^{3 / 2}\left(\delta t+h^{l}\right)+c_{2} \delta t\left(\delta t^{2}+h^{l+1}\right) .
$$

En appliquant le lemme de Gronwall discret on obtient :

$$
\left\|\delta_{t} e_{h}\right\|_{l^{\infty}\left(\|\cdot\|_{*}\right)}+\left\|\delta_{t} e_{h}\right\|_{l^{2}\left(\mathrm{~L}^{2}(\Omega)^{d}\right)} \leq c_{1} \delta t^{3 / 2}\left(\delta t+h^{l}\right)+c_{2} \delta t\left(\delta t^{2}+h^{l+1}\right),
$$

ce qui complète la preuve.

Remarque 4.3. Noter qu'il y a dans la majoration (4.10) un défaut d'optimalité d'un facteur $\delta t^{1 / 2}$. En effet, puisqu'on a montré dans le théorème 4.1 que l'erreur sur la vitesse en norme $l^{2}\left(\mathrm{~L}^{2}(\Omega)^{d}\right)$ est en $\delta t^{2}$, on pourrait s'attendre à une estimation sur l'incrément de l'erreur en norme $l^{2}\left(\mathrm{~L}^{2}(\Omega)^{d}\right)$ en $\delta t^{3}$. Ce défaut d'optimalité est due au manque d'optimalité de la majoration

$$
\left\|\delta_{t} e-\delta_{t} \tilde{e}_{h}\right\|_{l^{2}\left(\mathrm{~L}^{2}(\Omega)^{d}\right)} \leq c \delta t^{3 / 2}\left(\delta t+h^{l}\right)
$$

qui a été obtenue au lemme 3.3. Il est peut-être possible de retrouver une majoration optimale en travaillant sur les incréments des incréments. Le défaut d'optimalité subi à ce niveau est responsable de la perte d'un facteur $\delta t^{1 / 4}$ sur l'évaluation de l'erreur sur la vitesse en norme $l^{\infty}\left(\mathrm{L}^{2}(\Omega)^{d}\right)$ obtenue ci-dessous.

La majoration du lemme 4.7 permet maintenant d'établir le résultat majeur de cette section :

Théorème 4.2. Sous les hypothèses du théorème 3.2 et les hypothèses (H5-H6), on a :

$$
\left\|u-u_{h}\right\|_{l^{\infty}\left(\mathrm{L}^{2}(\Omega)^{d}\right)}+\left\|u-\tilde{u}_{h}\right\|_{l^{\infty}\left(\mathrm{L}^{2}(\Omega)^{d}\right)} \leq c\left(\delta t^{7 / 4}+\delta t^{3 / 4} h^{l}+h^{l+1}\right) .
$$


Preuve. On prend l'équation de conservation de quantité de mouvement (4.6) comme point de départ. On fait le produit scalaire de cette équation par $4 \delta t S_{h} \delta_{t} e_{h}^{k+1}$. En utilisant la symétrie de la forme bilinéaire $\left(v_{h}, S_{h} w_{h}\right)$ (cf. lemme 4.1) on obtient

$$
5\left\|\delta_{t} e_{h}^{k+1}\right\|_{*}^{2}+\left\|\delta_{t t} e_{h}^{k+1}\right\|_{*}^{2}+4 \delta t\left(A_{h} \tilde{e}_{h}^{k+1}, S_{h} \delta_{t} e_{h}^{k+1}\right)=\left\|\delta_{t} e_{h}^{k}\right\|_{*}^{2}+4 \delta t\left\langle R_{0}^{k+1}+R_{n l}^{k+1}, S_{h} \delta_{t} e_{h}^{k+1}\right\rangle .
$$

On utilise maintenant le lemme 4.4 en remarquant que $\delta_{t} e_{h}^{k+1}=P_{H_{h}} i_{h} \delta_{t} \tilde{e}_{h}^{k+1}$.

$$
\begin{aligned}
5\left\|\delta_{t} e_{h}^{k+1}\right\|_{*}^{2}+\left\|\delta_{t t} e_{h}^{k+1}\right\|_{*}^{2} & +4 \delta t\left(e_{h}^{k+1}, \delta_{t} e_{h}^{k+1}\right) \leq\left\|\delta_{t} e_{h}^{k}\right\|_{*}^{2}+c \delta t\left\|\delta_{t} e_{h}^{k+1}\right\|_{0}\left\|\tilde{e}_{h}^{k+1}-e_{h}^{k+1}\right\|_{0} \\
& +\gamma\left\|S_{h} \delta_{t} e_{h}^{k+1}\right\|_{1}^{2}+c_{\gamma} \delta t^{2} \sup _{v_{h} \in X_{h},\left\|v_{h}\right\|_{1}=1}\left\langle R_{0}^{k+1}+R_{n l}^{k+1}, v_{h}\right\rangle^{2},
\end{aligned}
$$

où la constante $\gamma \geq 0$ peut être choisie suffisamment petite de telle sorte que $\gamma\left\|S_{h} \delta_{t} e_{h}^{k+1}\right\|_{1}^{2} \leq\left\|\delta_{t} e_{h}^{k+1}\right\|_{*}^{2}$. La majoration du résidu est fournie par le lemme 4.5. Finalement on a

$$
\begin{aligned}
4\left\|\delta_{t} e_{h}^{k+1}\right\|_{*}^{2}+2 \delta t\left\|e_{h}^{k+1}\right\|_{0}^{2} & \leq\left\|\delta_{t} e_{h}^{k}\right\|_{*}^{2}+2 \delta t\left\|e_{h}^{k}\right\|_{0}^{2}+c \delta t\left\|\delta_{t} e_{h}^{k+1}\right\|_{0}\left\|\tilde{e}_{h}^{k+1}-e_{h}^{k+1}\right\|_{0} \\
& +c \delta t^{2}\left(\delta t^{2}+h^{l+1}+\left\|e_{h}^{k-1}\right\|_{0}+\left\|e_{h}^{k}\right\|_{0}+\left\|e_{h}^{k+1}\right\|_{0}\right)^{2}
\end{aligned}
$$

En prenant la somme de $k=1$ à $n \leq K-1$ on obtient

$$
\begin{aligned}
2 \delta t\left\|e_{h}^{n+1}\right\|_{0}^{2} & \leq\left\|\delta_{t} e_{h}^{1}\right\|_{*}^{2}+2 \delta t\left\|e_{h}^{1}\right\|_{0}^{2}+c_{1}\left\|e_{h}\right\|_{l^{2}\left(\mathrm{~L}^{2}(\Omega)^{d}\right)}\left\|\delta_{t} e_{h}\right\|_{l^{2}\left(\mathrm{~L}^{2}(\Omega)^{d}\right)} \\
& +c_{2} \delta t\left(\delta t^{2}+h^{l+1}\right)^{2}+c_{3} \delta t\left\|e_{h}\right\|_{l^{2}\left(\mathrm{~L}^{2}(\Omega)^{d}\right)} .
\end{aligned}
$$

L'hypothèse d'initialisation (H5), la majoration du lemme 4.7 et le résultat de convergence du théorème 4.1 donnent finalement

$$
\begin{aligned}
\left\|e_{h}^{n+1}\right\|_{0}^{2} & \leq c_{1}\left(\delta t^{2}+h^{l+1}\right)^{2}+c_{2}\left[\delta t^{3 / 2}\left(\delta t+h^{l}\right)+\delta t\left(\delta t^{2}+h^{l+1}\right)\right]\left(\delta t+h^{l}\right), \\
& \leq c_{1}\left(\delta t^{2}+h^{l+1}\right)^{2}+c_{2} \delta t^{3 / 2}\left(\delta t+h^{l}\right)^{2} .
\end{aligned}
$$

ce qui complète la preuve.

Remarque 4.4. L'exposant $7 / 4$ n'est probablement pas optimal pour la convergence en norme $l^{\infty}\left(\mathrm{L}^{2}(\Omega)^{d}\right)$. Il est peut-être possible d'améliorer un peu le résultat en invoquant des arguments techniques un peu plus sophistiqués sur les incréments d'ordre deux.

\subsection{Une estimation en norme faible sur la pression}

Il est possible d'améliorer un peu le résultat de convergence obtenu sur la pression dans le théorème 3.2 en introduisant une norme faible dépendente du maillage. Pour tout $q$ dans $\mathrm{L}^{2}(\Omega)$ on définit :

$$
\|q\|_{B_{h}, A_{h}}=\sup _{v_{h} \in X_{h}} \frac{\left(q, \nabla \cdot v_{h}\right)}{\left\|A_{h}^{\mathrm{t}} v_{h}\right\|_{0}} .
$$

Grâce à la condition "inf-sup," il est clair que la restriction de $\|\cdot\|_{B_{h}, A_{h}}$ à $M_{h}$ définit une norme, toutefois, cette norme est plus faible que la norme $\|\cdot\|_{0}$. Plus précisement, la nouvelle norme est la contrepartie discrète sur $M_{h}$ de la norme

$$
\|q\|_{\nabla \cdot, \nabla^{2}}=\sup _{v \in \mathrm{H}_{0}^{1}(\Omega)^{d} \cap \mathrm{H}^{2}(\Omega)^{d}} \frac{(q, \nabla \cdot v)}{\left\|v_{h}\right\|_{2}} .
$$


Grâce aux propriétés régularisantes de l'opérateur de Stokes, il est possible de montrer le résultat suivant

$$
\forall q \in \mathrm{L}_{0}^{2}(\Omega), \quad c_{1}\|q\|_{-1} \leq\|q\|_{\nabla \cdot, \nabla^{2}} \leq c_{2}\|q\|_{0},
$$

qui signifie que la topologie associée à la nouvelle norme est plus grossière que celle de $\mathrm{L}^{2}(\Omega)$ mais qu'elle est plus fine que celle de $\mathrm{H}^{-1}(\Omega)$.

Avec la nouvelle norme discrète pour mesurer l'erreur sur la pression, on a le résultat suivant

Théorème 4.3. Sous les hypothèses du théorème 3.2 et les hypothèses (H5-H6), on a :

$$
\left\|p-p_{h}\right\|_{l^{2}\left(\|\cdot\|_{B_{h}, A_{h}}\right)} \leq c\left(\delta t^{3 / 2}+h^{l}\right) .
$$

Preuve. D'après (3.13) on a

$$
\left\|\epsilon_{h}^{k+1}\right\|_{B_{h}, A_{h}} \leq \frac{3\left\|\delta_{t} e_{h}^{k+1}\right\|_{0}}{2 \delta t}+\frac{\left\|\delta_{t} e_{h}^{k}\right\|_{0}}{2 \delta t}+\left\|\tilde{e}_{h}^{k+1}\right\|_{0}+\sup _{v_{h} \in X_{h},\left\|v_{h}\right\|_{1}=1}\left\langle R_{0}^{k+1}+R_{n l}^{k+1}, v_{h}\right\rangle .
$$

Le résultat cherché est une conséquence de cette inégalité, des majorations des lemmes 4.5 et 4.7 et du théorème 4.1 .

Remarque 4.5. La difficultés qu'on rencontre à établir des résultats de convergence à l'ordre deux (ou en tous cas à un ordre plus grand que un) dans des normes naturelles pour la pression (i.e. dans $\mathrm{L}^{2}(\Omega)$ ) ou pour la vitesse (i.e. dans $\mathrm{H}^{1}(\Omega)^{d}$ ) sont symptomatiques de la présence d'une couche limite numérique à la frontière de $\Omega$. Cette couche limite est imperceptible à l'ordre deux pour la vitesse mesurée dans la norme $\mathrm{L}^{2}(\Omega)^{d}$, en revanche elle apparaît à un ordre compris entre un et deux lorsque la vitesse est mesurée dans la norme $\mathrm{H}^{1}(\Omega)^{d}$ ou lorsque la pression est mesurée dans la norme $L^{2}(\Omega)$ (voir Rannacher [19] pour une discussion détaillée sur la couche limite numérique induite par le schéma non incrémental continu en espace).

\section{Remarques Sur L'ERREUR DE FRACTIONNEMENT}

On finit cet article par une étude de l'erreur de fractionnement des algorithmes de projection basés sur une correction de pression incrémentale. Le résultat essentiel de cette section est que l'erreur de fractionnement est d'ordre deux pour la vitesse dans la norme $l^{2}\left(\mathrm{~L}^{2}(\Omega)^{d}\right)$.

Pour illustrer cette propriété remarquable, considérons l'algorithme de projection incrémental basé sur une approximation par différentiation rétrograde d'ordre un de la dérivée temporelle. En conservant les mêmes notations que dans les sections précédentes, on considère l'algorithme suivant : pour $1 \leq k \leq K-1$ on cherche $\tilde{u}_{h}^{k+1}$ dans $X_{h}$ tel que :

$$
\frac{\tilde{u}_{h}^{k+1}-i_{h}^{\mathrm{t}} u_{h}^{k}}{\delta t}+A_{h} \tilde{u}_{h}^{k+1}+D_{h}\left(\tilde{u}_{h}^{k}, \tilde{u}_{h}^{k+1}\right)=f_{h}^{k+1}-B_{h}^{\mathrm{t}} p_{h}^{k} .
$$

On cherche ensuite $u_{h}^{k+1}$ dans $Y_{h}$ et $p_{h}^{k+1}$ dans $M_{h}$ tel que

$$
\left\{\begin{array}{l}
\frac{u_{h}^{k+1}-i_{h} \tilde{u}_{h}^{k+1}}{\delta t}+C_{h}^{\mathrm{t}}\left(p_{h}^{k+1}-p_{h}^{k}\right)=0, \\
C_{h} u_{h}^{k+1}=0 .
\end{array}\right.
$$

Bien sûr, la mise en œuvre pratique de cet algorithme se fait en éliminant la vitesse projetée comme expliqué dans la remarque 2.2 . 
Considérons maintenant l'algorithme totalement couplé : trouver $w_{z, h}^{k+1}$ dans $X_{h}$ et $q_{z, h}^{k+1}$ dans $M_{h}$ tels que :

$$
\left\{\begin{array}{l}
\frac{w_{z, h}^{k+1}-w_{z, h}^{k}}{\delta t}+A_{h} w_{z, h}^{k+1}+D_{h}\left(w_{z, h}^{k}, w_{z, h}^{k+1}\right)+B_{h}^{\mathrm{t}} q_{z, h}^{k+1}=f_{h}^{k+1} \\
B_{h} w_{z, h}^{k+1}=0 .
\end{array}\right.
$$

La différence entre $w_{z, h}^{k+1}$ et $\tilde{u}_{h}^{k+1}$ ou bien entre $w_{z, h}^{k+1}$ et $u_{h}^{k+1}$ est par définition l'erreur de fractionnement. C'est l'erreur induite par le découplage, opéré dans l'algorithme de projection, entre la contrainte d'incompressibilité et les phénomènes de convection-diffusion. La caractéristique remarquable de la techniques incrémentale (i.e. celle pour laquelle la pression est rendue explicite à l'étape de convection-diffusion) est résumée par le résultat suivant :

Théorème 5.1. Il existe une constante c qui ne dépend que de $\left(w_{z, h}, q_{z, h}\right)$ et $T$, tel que

$$
\left\|w_{z, h}-u_{h}\right\|_{l^{2}\left(\mathrm{~L}^{2}(\Omega)^{d}\right)}+\left\|w_{z, h}-\tilde{u}_{h}\right\|_{l^{2}\left(\mathrm{~L}^{2}(\Omega)^{d}\right)} \leq c \delta t^{2} .
$$

Preuve. On ne donne ici qu'une idée de la preuve. Introduisons les notations suivantes pour désigner les erreurs de fractionnement : $\tilde{e}_{z, h}^{k}=w_{z, h}^{k}-\tilde{u}_{h}^{k}, e_{z, h}^{k}=w_{z, h}^{k}-u_{h}^{k}$ et $\epsilon_{z, h}^{k}=q_{z, h}^{k}-p_{h}^{k}$. Il est clair que les erreurs de fractionnement sont contrôlées par les systèmes d'équations

$$
\begin{gathered}
\frac{\tilde{e}_{z, h}^{k+1}-i_{h}^{\mathrm{t}} e_{z, h}^{k}}{\delta t}+A_{h} \tilde{e}_{z, h}^{k+1}=-B_{h}^{\mathrm{t}} \psi_{z, h}^{k}+R_{z, h}^{k+1}\left(\tilde{e}_{z, h}^{k}, \tilde{e}_{z, h}^{k+1}\right), \\
\left\{\begin{array}{l}
\frac{e_{z, h}^{k+1}-i_{h} \tilde{e}_{z, h}^{k+1}}{\delta t}+C_{h}^{\mathrm{t}}\left(\epsilon_{z, h}^{k+1}-\psi_{z, h}^{k}\right)=0, \\
C_{h} e_{z, h}^{k+1}=0,
\end{array}\right.
\end{gathered}
$$

où on a posé $\psi_{z, h}^{k}=q_{z, h}^{k+1}-p_{h}^{k}=\delta_{t} q_{z, h}^{k+1}+\epsilon_{z, h}^{k}$ et le reste non linéaire est défini par

$$
R_{z, h}^{k+1}\left(\tilde{e}_{z, h}^{k}, \tilde{e}_{z, h}^{k+1}\right)=D_{h}\left(w_{z, h}^{k}, w_{z, h}^{k+1}\right)-D_{h}\left(\tilde{u}_{h}^{k}, \tilde{u}_{h}^{k+1}\right)
$$

La structure de ces équations est quasiment la même que (3.9)-(3.10) sauf que la seule source d'erreur qui subsite maintenant est le terme $B_{h}^{\mathrm{t}} \psi_{z, h}^{k}$ qui est au second membre du pas de convection-diffusion. On peut alors reproduire tous les arguments des sections 3 et 4.

Le résultat un peu suprenant du théorème 5.1 peut être vérifié numériquement assez facilement. Il suffit pour un problème donné de réaliser une marche en temps en évaluant à chaque pas de temps la solution du système couplé (5.3) et la solution du système fractionné (5.1)-(5.2). La solution du sytème couplée peut être calculée de multiples façons (résolution directe ou itérative de l'opérateur d'Uzawa, technique de matrice d'influence, etc.) mais en pratique son évaluation est toujours beaucoup plus coûteuse que celle de la solution découplée (des rapports 20 à 50 en temps de calcul sont typiques). Cette expérience a été réalisée dans [14] pour le problème de la cavité entraînée en dimension 2 en utilisant des éléments finis $P_{2} / P_{1}$. L'expérience numérique rapportée dans [14] montre clairement que l'erreur de fractionnement est bien d'ordre deux conformément à l'énoncé du théorème 5.1 . 


\section{REFERENCES}

[1] C. Amrouche and V. Girault, On the existence and regularity of the solution of Stokes problem in arbitrary dimension. Proc. Japan Acad. 67 (1991) 171-175.

[2] I. Babǔska, The finite element method with Lagragian multipliers. Numer. Math. 20 (1973) 179-192.

[3] C. Bernardi and G. Raugel, A conforming finite element method for the time-dependent Navier-Stokes equations. SIAM J. Numer. Anal. 22 (1985) 455-473.

[4] H. Brezis, Analyse fonctionnelle, théorie et applications. Masson, Paris (1983).

[5] F. Brezzi, On the existence uniqueness and approximation of saddle-point problems arising from Lagrangian multipliers. RAIRO R2 (1974) 129-151.

[6] L. Cattabriga, Su un problema al contorno relativo al sistema di equazioni di Stokes. Rend. Sem. Mat. Univ. Padova 31 (1961) 308-340.

[7] A.J. Chorin, Numerical solution of the Navier-Stokes equations. Math. Comp. 22 (1968) 745-762.

[8] A. J. Chorin, On the convergence of discrete approximations to the Navier-Stokes equations. Math. Comp. 23 (1969) $341-353$.

[9] V. Girault and P.-A. Raviart, Finite Element Methods for Navier-Stokes Equations. Springer Series in Computational Mathematics 5 Springer-Verlag (1986).

[10] K. Goda, A multistep technique with implicit difference schemes for calculating two- or three-dimensional cavity flows. $J$. Comput. Phys. 30 (1979) 76-95.

[11] J.-L. Guermond, Some practical implementations of projection methods for Navier-Stokes equations. RAIRO Modél. Math. Anal. Numér. 30 (1996) 637-667.

[12] J.-L. Guermond, Sur l'approximation des équations de Navier-Stokes instationnaires par une méthode de projection. C. R. Acad. Sci. Paris 319 (1994) 887-892.

[13] J.-L. Guermond and L. Quartapelle, Unconditionally stable Finite-Element Method for the unsteady Navier-Stokes equations, 9th International Conference on Finite Element in Fluids. Venezia, Italy, October 1995 I 367-376.

[14] J.-L. Guermond and L. Quartapelle, Calculation of incompressible viscous flows by an unconditionally stable projection finite element method. J. Comput. Phys. 132 (1997) 12-33.

[15] J.-L. Guermond and L. Quartapelle, On the approximation of the Navier-Stokes equations by finite element projection methods. Numer. Math. 80 (1998) 207-238.

[16] J.G. Heywood and R. Rannacher, Finite element approximation of the nonstationary Navier-Stokes problem, I, II, III, and IV. SIAM J. Numer. Anal. 19 (1982) 275-311; 23 (1986) 750-777; 25 (1988) 489-512; 27 (1990) 353-384.

[17] L. Quartapelle, Numerical Solution of the Incompressible Navier-Stokes Equations. ISNM 113 Birkhäuser, Basel (1993).

[18] A. Quarteroni and A. Valli, Numerical Approximation of Partial Differential Equations. Springer Series in Computational Mathematics, Vol. 5, Springer-Verlag (1994).

[19] R. Rannacher, On Chorin's projection method for the incompressible Navier-Stokes equations. Lectures Notes in Mathematics Springer, Berlin (1992) 167-183.

[20] R. Temam, Navier-Stokes Equations. Studies in Mathematics and its Applications, Vol. 2. North-Holland (1977).

[21] R. Temam, Une méthode d'approximation de la solution des équations de Navier-Stokes. Bull. Soc. Math. France 98 (1968) 115-152.

[22] J. Van Kan, A second-order accurate pressure-correction scheme for viscous incompressible flow. SIAM J. Sci. Stat. Comput. 7 (1986) 870-891. 